\title{
Global HCFC-22 measurements with MIPAS: retrieval, validation, global distribution and its evolution over 2005-2012
}

\author{
M. Chirkov ${ }^{1}$, G. P. Stiller ${ }^{1}$, A. Laeng ${ }^{1}$, S. Kellmann ${ }^{1}$, T. von Clarmann ${ }^{1}$, C. D. Boone ${ }^{2}$, J. W. Elkins ${ }^{3}$, A. Engel ${ }^{4}$, \\ N. Glatthor ${ }^{1}$, U. Grabowski ${ }^{1}$, C. M. Harth ${ }^{5}$, M. Kiefer ${ }^{1}$, F. Kolonjari ${ }^{6}$, P. B. Krummel ${ }^{7}$, A. Linden ${ }^{1}$, C. R. Lunder $^{8}$, \\ B. R. Miller ${ }^{3}$, S. A. Montzka ${ }^{3}$, J. Mühle ${ }^{5}$, S. O'Doherty ${ }^{9}$, J. Orphal ${ }^{1}$, R. G. Prinn ${ }^{10}$, G. Toon ${ }^{11}$, M. K. Vollmer ${ }^{12}$, \\ K. A. Walker ${ }^{2,6}$, R. F. Weiss ${ }^{5}$, A. Wiegele ${ }^{1}$, and D. Young ${ }^{9}$ \\ ${ }^{1}$ Karlsruhe Institute of Technology (KIT), Institute for Meteorology and Climate Research (IMK), Karlsruhe, Germany \\ ${ }^{2}$ University of Waterloo, Department of Chemistry, Waterloo, Ontario, Canada \\ ${ }^{3}$ NOAA/ESRL Climate Monitoring Division, Boulder, Colorado, USA \\ ${ }^{4}$ Goethe-Universität Frankfurt, Experimental Atmospheric Research Institute for Atmospheric and Environmental Sciences, \\ Frankfurt, Germany \\ ${ }^{5}$ Scripps Institution of Oceanography, University of California, San Diego, La Jolla, California, USA \\ ${ }^{6}$ University of Toronto, Department of Physics, Toronto, Ontario, Canada \\ ${ }^{7}$ CSIRO Oceans \& Atmosphere Flagship, Aspendale, Victoria, Australia \\ ${ }^{8}$ Norwegian Institute for Air Research, Kjeller, Norway \\ ${ }^{9}$ Atmospheric Chemistry Research Group, School of Chemistry, University of Bristol, Bristol, UK \\ ${ }^{10}$ Center for Global Change Science, MIT, Cambridge, MA, USA \\ ${ }^{11}$ Jet Propulsion Laboratory and California Institute of Technology, Pasadena, California, USA \\ ${ }^{12}$ Laboratory for Air Pollution and Environmental Technology, Empa, Swiss Federal Laboratories for Materials Science and \\ Technology, Dübendorf, Switzerland
}

Correspondence to: G. P. Stiller (gabriele.stiller@kit.edu)

Received: 10 April 2015 - Published in Atmos. Chem. Phys. Discuss.: 27 May 2015

Revised: 25 February 2016 - Accepted: 1 March 2016 - Published: 15 March 2016

\begin{abstract}
We report on HCFC-22 data acquired by the Michelson Interferometer for Passive Atmospheric Sounding (MIPAS) in the reduced spectral resolution nominal observation mode. The data cover the period from January 2005 to April 2012 and the altitude range from the upper troposphere (above cloud top altitude) to about $50 \mathrm{~km}$. The profile retrieval was performed by constrained nonlinear least squares fitting of modelled spectra to the measured limb spectral radiances. The spectral $v_{4}$-band at $816.5 \pm 13 \mathrm{~cm}^{-1}$ was used for the retrieval. A Tikhonov-type smoothing constraint was applied to stabilise the retrieval. In the lower stratosphere, we find a global volume mixing ratio of HCFC22 of about 185 pptv in January 2005. The rate of linear growth in the lower latitudes lower stratosphere was about 6 to 7 pptv year $^{-1}$ in the period 2005-2012. The profiles obtained were compared with ACE-FTS satellite data v3.5, as well as with MkIV balloon profiles and cryosampler balloon
\end{abstract}

measurements. Between 13 and $22 \mathrm{~km}$, average agreement within -3 to +5 pptv (MIPAS - ACE) with ACE-FTS v3.5 profiles is demonstrated. Agreement with MkIV solar occultation balloon-borne measurements is within 10-20 pptv below $30 \mathrm{~km}$ and worse above, while in situ cryosampler balloon measurements are systematically lower over their full altitude range by $15-50$ pptv below $24 \mathrm{~km}$ and less than 10 pptv above $28 \mathrm{~km}$. MIPAS HCFC-22 time series below $10 \mathrm{~km}$ altitude are shown to agree mostly well to corresponding time series of near-surface abundances from the NOAA/ESRL and AGAGE networks, although a more pronounced seasonal cycle is obvious in the satellite data. This is attributed to tropopause altitude fluctuations and subsidence of polar winter stratospheric air into the troposphere. A parametric model consisting of constant, linear, quasibiennial oscillation (QBO) and several sine and cosine terms with different periods has been fitted to the temporal varia- 
tion of stratospheric HCFC-22 for all $10^{\circ}$-latitude/1-to-2-kmaltitude bins. The relative linear variation was always positive, with relative increases of $40-70 \%$ decade $^{-1}$ in the tropics and global lower stratosphere, and up to $120 \%$ decade $^{-1}$ in the upper stratosphere of the northern polar region and the southern extratropical hemisphere. Asian HCFC-22 emissions have become the major source of global upper tropospheric HCFC-22. In the upper troposphere, monsoon air, rich in HCFC-22, is instantaneously mixed into the tropics. In the middle stratosphere, between 20 and $30 \mathrm{~km}$, the observed trend is inconsistent with the trend at the surface (corrected for the age of stratospheric air), hinting at circulation changes. There exists a stronger positive trend in HCFC-22 in the Southern Hemisphere and a more muted positive trend in the Northern Hemisphere, implying a potential change in the stratospheric circulation over the observation period.

\section{Introduction}

HCFC-22 $\left(\mathrm{CHClF}_{2}\right)$ is a chlorine source gas and a greenhouse gas (IPCC, 2014). The sources of HCFC-22 are anthropogenic emissions due to its use as a propellant and refrigerant. The gas is removed from the atmosphere by photolysis and by reactions with $\mathrm{O}\left({ }^{1} \mathrm{D}\right), \mathrm{Cl}^{-}$, and the $\mathrm{OH}$ radical. The chemical lifetime of HCFC-22 in the stratosphere is 165 years (12 years for its global total atmospheric lifetime), according to SPARC (2013), but has been estimated to a significantly longer span of $260 \pm 25$ years by Moore and Remedios (2008). The radiative forcing potential of HCFC22 is $0.208 \mathrm{~W} \mathrm{~m}^{-2} \mathrm{ppbv}^{-1}$, and its ozone depletion potential is about 20 times lower than that of CFC-12 (0.04) (WMO, 2014). Production and import of HCFC-22 is limited by the Montreal Protocol on Substances that Deplete the Ozone Layer and will be banned by 2030 from dispersive uses in developed countries (United Nations Environment Programme, 2009). The 2007 Adjustment to the Protocol asks for a $100 \%$ reduction by 2030 for all countries, including developing nations - albeit with a $2.5 \%$ allowance for servicing of refrigeration and air conditioning equipment existing on 1 January 2030 for the period 2030-2040 and subject to review in 2015. Therefore the overall reduction shall already be 97.5 $100 \%$ by 2030 .

Ambient HCFC-22 was first measured by Rasmussen et al. (1980) by air sampling techniques. Atmospheric HCFC-22 abundances are typically measured on site by gas-chromatographic techniques or by collecting samples in flasks or by balloon-borne air-sampling measurements (Engel et al., 1997) followed by subsequent gaschromatographic analysis in a central laboratory (Montzka et al., 2009; O’Doherty et al., 2004; Yokouchi et al., 2006). Further, there exist remote measurements by infrared spectroscopy from ground-based (Rinsland et al., 2005b; Zander et al., 2005; Gardiner et al., 2008), balloon-borne (Mur- cray et al., 1975; Williams et al., 1976; Goldman et al., 1981), or space-borne (Zander et al., 1987; Rinsland et al., 2005a; Moore and Remedios, 2008) platforms in solar absorption geometry. Among recently flying space-borne instruments, only the Atmospheric Chemistry Experiment Fourier Transform Spectrometer (ACE-FTS; solar occultation) and the Michelson Interferometer for Passive Atmospheric Sounding (MIPAS; limb emission) have been providing measurements of HCFC-22 (Kolonjari et al., 2012; Park et al., 2014; Moore and Remedios, 2008). The history of measurements is summarised in the study by von Clarmann (2013).

In this paper we present and discuss HCFC-22 distributions and time series as retrieved with the MIPAS data processor developed and operated by the Institute for Meteorology and Climate Research at the Karlsruhe Institute of Technology (KIT-IMK) in Germany in cooperation with the Instituto de Astrofísica de Andalucía (IAA, CSIC) in Granada, Spain. In the next section we provide a description of the MIPAS instrument and measurements. The retrieval strategy and error estimation are summarised in Sect. 3. Section 4 reports on the validation of this data set, and Sect. 5 presents global distributions of HCFC-22 and an assessment of temporal variations including a linear trend. The derived estimates of trends will be compared to those from long-term surface data records. Section 6 contains the discussion of the results and the summary.

\section{MIPAS data}

MIPAS measured the thermal emission of the atmosphere, and thus provided data during day and night. It was a cryogenic limb-emission Fourier transform spectrometer (FTS) designed for measurement of trace species from space (European Space Agency, 2000; Endemann and Fischer, 1993; Endemann et al., 1996; Fischer and Oelhaf, 1996; Fischer et al., 2008). MIPAS was 1 of 10 instruments aboard the Environmental Satellite (Envisat). Envisat was launched into a sunsynchronous polar orbit on 1 March 2002 at approximately $800 \mathrm{~km}$, with an orbital period of about $101 \mathrm{~min}$, resulting in more than 14 orbits per day. The end of the Envisat mission was declared on 9 May 2012 after loss of communication with the satellite on 8 April 2012.

MIPAS sounded the atmosphere tangentially to the Earth in the infrared spectral range $(4.15-14.6 \mu \mathrm{m})$ covering tangent altitudes from about 7 to $72 \mathrm{~km}$ in its nominal observation mode. The instrument's field of view was approximately $3 \mathrm{~km}$ (vertically) $\times 30 \mathrm{~km}$ (horizontally). MIPAS operated from July 2002 to March 2004 with full spectral resolution as specified: $0.05 \mathrm{~cm}^{-1}$ in terms of full width at half maximum, after apodisation with the "strong" function suggested by Norton and Beer (1976). The full resolution mode was stopped in March 2004. Starting from January 2005 and up to the end of the mission, the spectral resolution of MIPAS 
was degraded from 0.05 to $0.12 \mathrm{~cm}^{-1}$ (apodised). These later measurements are referred to as "reduced resolution mode".

The data analysis reported in this paper relies on the ESAprovided so-called level- $1 \mathrm{~b}$ data product which includes calibrated phase-corrected and geolocated radiance spectra (Nett et al., 1999). The versions of ESA level-1b data used are IPF (Instrument Processor Facility) 5.02-5.06. All spectra under consideration here were recorded according to the nominal reduced resolution measurement mode, including per limb sequence 27 tangent altitudes between about 7 and $72 \mathrm{~km}$, with the tangent altitude adjustment following roughly the tropopause altitude over latitudes. The vertical distance between adjacent tangent heights varies between $1.5 \mathrm{~km}$ in the upper troposphere/lower stratosphere up to $4.5 \mathrm{~km}$ in the mesosphere. One limb scan is recorded per each 410 increment along the polar sun-synchronous orbit, leading to a dense horizontal sampling which is independent of the season or latitude band. As a result, MIPAS covers all latitudes during one orbit.

\section{Retrieval}

The retrieval of HCFC-22 profiles presented here was performed with a MIPAS data processor dedicated for research applications, which has been developed at the Institut für Meteorologie und Klimaforschung (IMK) in cooperation with the Instituto de Astrofísica de Andalucía (IAA). The IMK retrieval processor consists of the radiative transfer algorithm KOPRA (Karlsruhe Optimized and Precise Radiative transfer Algorithm) (Stiller, 2000) and the retrieval algorithm RCP (retrieval control program). Local spherical homogeneity of the atmosphere is assumed here, i.e. atmospheric state parameters related to one limb sounding sequence are assumed not to vary with latitude or longitude but only with altitude. An exception is temperature for which horizontal gradients are considered in the retrieval (Kiefer et al., 2010). Although the retrieval processor used supports the treatment of nonlocal thermodynamic equilibrium (non-LTE), LTE has been assumed because it is a good enough approximation in the altitude range under consideration.

The general strategy of the IMK/IAA data processing has been documented in von Clarmann et al. (2003).

\subsection{Retrieval of HCFC-22}

HCFC-22 is retrieved by a constrained multi-parameter nonlinear least-squares fitting of modelled to measured spectra. Spectral data from all tangent altitudes are analysed within one inversion process, as suggested by Carlotti (1988). Volume mixing ratio (vmr) vertical profiles are retrieved on a fixed, i.e. tangent height independent altitude grid which is finer than the tangent height spacing (1-km steps from 4 to $35 \mathrm{~km}$; then $5-\mathrm{km}$ grid width from 35 to $50 \mathrm{~km} ; 10-\mathrm{km}$ grid width from 50 to $100 \mathrm{~km} ; 120 \mathrm{~km}$ ). In order to ob-

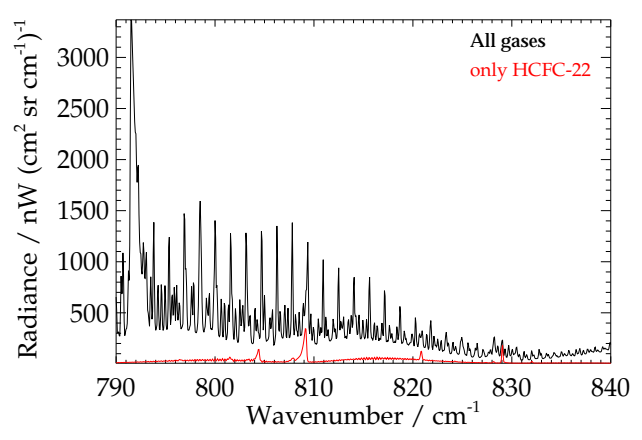

Figure 1. Typical spectrum of HCFC-22 (red curve) and combined spectrum of 24 gases (HCFC-22 included) (black curve) at $16 \mathrm{~km}$ tangent altitude.

tain stable profiles, the profiles have been constrained such that the first-order finite difference quotient $\Delta \mathrm{vmr} / \Delta$ altitude at adjacent altitude grid points was minimised, similar as proposed by Tikhonov (1963). An altitude-constant profile of zero mixing ratio throughout was taken as a priori, while the initial guess profile was from the MIPAS climatology (Kiefer et al., 2002). For the retrieval of HCFC22, we have used four microwindows of the MIPAS spectrum $\left(803.500\right.$ to $804.750 \mathrm{~cm}^{-1}, 808.250$ to $809.750 \mathrm{~cm}^{-1}$, 820.500 to $821.125 \mathrm{~cm}^{-1}$ and 828.750 to $829.500 \mathrm{~cm}^{-1}$ ). For HCFC-22, the HITRAN2K spectroscopic database (Rothman et al., 2003) was used, with some updates for interfering species.

Figure 1 shows the atmospheric limb-emission radiance spectrum for mid-latitudes at $16 \mathrm{~km}$ tangent altitude including contributions from $\mathrm{H}_{2} \mathrm{O}, \mathrm{CO}_{2}, \mathrm{O}_{3}, \mathrm{NO}_{2}, \mathrm{NH}_{3}, \mathrm{HNO}_{3}$, $\mathrm{ClO}, \mathrm{OCS}, \mathrm{HCN}, \mathrm{CH}_{3} \mathrm{Cl}, \mathrm{C}_{2} \mathrm{H}_{2}, \mathrm{C}_{2} \mathrm{H}_{6}, \mathrm{COF}_{2}, \mathrm{C}_{2} \mathrm{H}_{4}, \mathrm{HNO}_{4}$, CFC-11, CCl $4, \mathrm{CFC}-113, \mathrm{ClONO}_{2}, \mathrm{CH}_{3} \mathrm{CCl}_{3}, \mathrm{CH}_{3} \mathrm{OH}$, $\mathrm{C}_{2} \mathrm{H}_{3} \mathrm{NO}_{5}$ (peroxyacetyl nitrate, $\mathrm{PAN}$ ), $\mathrm{C}_{3} \mathrm{H}_{6} \mathrm{O}$ and HCFC22 (black curve). In addition, the sole contribution of HCFC22 is shown in red.

For some quantities, information from preceding retrieval steps was used for the retrieval of HCFC-22: a correction of the spectral shift caused by a less-than-perfect frequency calibration; the tangent altitudes of the limb measurements; temperature; and finally the mixing ratios of the species $\mathrm{O}_{3}, \mathrm{H}_{2} \mathrm{O}, \mathrm{HNO}_{3}, \mathrm{ClO}, \mathrm{ClONO}_{2}, \mathrm{CFC}-11, \mathrm{HNO}_{4}$ as well as $\mathrm{C}_{2} \mathrm{H}_{6}$. The vmrs of $\mathrm{CO}_{2}$ and all other remaining gases were taken from a climatological database, and the temperature was retrieved from $\mathrm{CO}_{2}$ lines (von Clarmann et al., 2003, 2009b).

Simultaneously with HCFC-22 and with adequate regularisation, we jointly fitted the following quantities: the mixing ratio of PAN; a wavenumber-independent continuum absorption coefficient per microwindow and altitude accounting for aerosol emission; and an additive radiative offset for each microwindow. The data versions are V5r_F22_220 and V5r_F22_221. The only difference between these versions is the source of temperature analysis data used as a priori for 


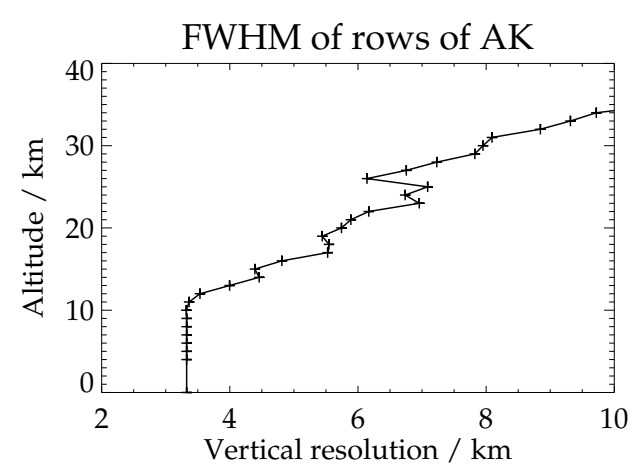

Figure 2. Altitude resolution in terms of full width at half maximum (FWHM) of a row of the averaging kernel matrix.

the preceding temperature retrieval. Results are equivalent and different version numbers are used only to guarantee full traceability of the retrievals.

\subsection{Diagnostics}

Diagnostic quantities to characterise the HCFC-22 measurements include estimates of measurement noise, of retrieval errors caused by uncertainties in ancillary parameters used in the radiative transfer modelling, and the averaging kernel (AK) matrix (Rodgers, 2000).

Table 1 shows the estimated total retrieval error of a HCFC-22 profile measured at $18.6^{\circ} \mathrm{S}$ and $111.6^{\circ} \mathrm{W}$ on 9 January 2009. This error estimate is considered to be roughly representative for the entire data set but is rather conservative, due to the typically low lower stratospheric temperatures at tropical latitudes, which are associated with a lower signal. The total error is the square root of the quadratic sum of noise error and parameter errors. Its most important components are the uncertainty of the elevation pointing of the line of sight (LOS), the uncertainty of pre-retrieved $\mathrm{O}_{3}$ mixing ratios, the gain calibration uncertainty, the residual spectral shift uncertainty, and the instrument line shape (ILS) uncertainty. The spectroscopic error (not reported in the table) is about $5 \%$. In the given altitude range, errors resulting from uncertainties of interfering species contribute to the total error by less than $1 \%$.

The percentage of non-converged profiles is about 0.01 to $0.02 \%$. The strength of the regularisation, i.e. the weight of the constraint, has been chosen to be altitude-dependent with a scheme proposed by Steck (2002) such that the retrieved profile represents approximately $5^{\circ}$ of freedom, corresponding to a typical altitude resolution of $3 \mathrm{~km}$ at $10 \mathrm{~km}$ height and $7 \mathrm{~km}$ at $30 \mathrm{~km}$ height, and further increasing with height (Fig. 2). Here the vertical resolution is provided in terms of full width at half maximum of a row of the averaging-kernel (AK) matrix. The rows of the AK-matrix show how much information from other atmospheric altitudes contribute to the vmr on the given retrieval altitude. An example of the rows of

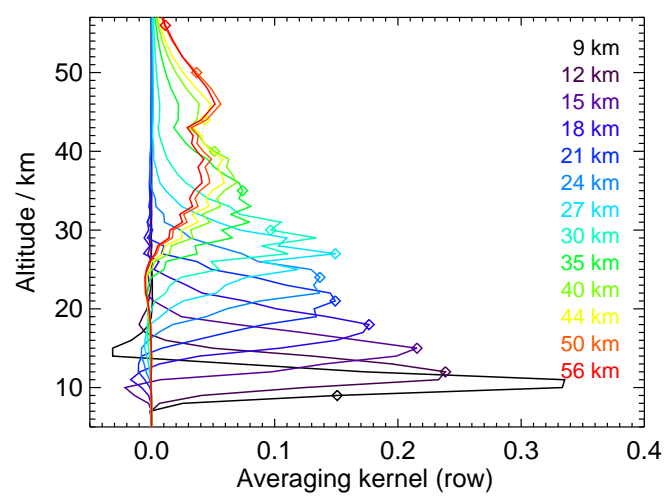

Figure 3. Rows of averaging kernel of HCFC-22 measurements for reduced spectral resolution nominal mode. The diamonds represent the nominal altitudes (e.g. the diagonal value of the averaging kernel matrix). For clarity, only every third kernel is shown.

the HCFC-22 averaging kernel matrix is shown in Fig. 3. The largest peaks of the averaging kernels are generally found in the upper troposphere. This is because the retrieval is more strongly regularised at higher altitudes. In general, the retrieval is well-behaved in a sense that the averaging kernels peak at or close to their nominal altitudes (marked by diamonds in Fig. 3) between 12 and about $35 \mathrm{~km}$. The integral over the averaging kernels in this altitude range are, at good accuracy, unity; thus our choice of the constraint does not impose any bias.

The information evaluated by a limb retrieval is not located in one single point but spread horizontally along the line of sight direction. The horizontal information smearing of the HCFC-22 measurement is estimated by using the method of von Clarmann et al. (2009a). In terms of full width at half maximum of the rows of the horizontal averaging kernel matrix, the horizontal information smearing of a MIPAS retrieval typically varies between about 210 and $680 \mathrm{~km}$ for most species, altitudes and atmospheric conditions. For HCFC-22 the horizontal information smearing, calculated as the half width of the horizontal component of the 2-D averaging kernel, is approximately $300 \mathrm{~km}$ at altitudes below $15 \mathrm{~km}, 608 \mathrm{~km}$ at altitude $20 \mathrm{~km}$ and approximately $550 \mathrm{~km}$ above (see Table 2).

The information displacement is defined as the horizontal distance between the point where the most information comes from and the nominal geolocation of the limb scan, which is defined as the geolocation of the tangent point of the middle line of sight in a MIPAS limb scan. The information displacement in case of HCFC-22 varies between $-121 \mathrm{~km}$ at $40 \mathrm{~km}$ altitude (the negative sign refers to displacement beyond the tangent point with respect to the satellite) and $133 \mathrm{~km}$ at $15 \mathrm{~km}$ altitude, and is lowest and positive (i.e. displacement towards the satellite) in the middle stratosphere (see Table 2). 
Table 1. Error budget of a V5r HCFC-22 (nominal mode, reduced resolution) retrieval on 9 January 2009 , $18.6^{\circ} \mathrm{S}$ latitude and $111.6^{\circ} \mathrm{W}$ longitude, orbit 35874 , for selected altitudes. The errors are given in units of mixing ratios (pptv), and additionally, in parentheses, in percentage units $(\%)$.

\begin{tabular}{rrrrrrrrr}
\hline $\begin{array}{r}\text { V5r } \\
\text { HCFC-22 } \\
\text { height }\end{array}$ & $\begin{array}{r}\text { Total } \\
\text { error }\end{array}$ & Noise & $\begin{array}{r}\text { Parameter } \\
\text { error }\end{array}$ & $\mathrm{O}_{3}$ & LOS & Shift & Gain & ILS \\
\hline $40 \mathrm{~km}$ & $14(17.3)$ & $13(16.1)$ & $5.7(7.0)$ & $0.3(0.3)$ & $1.7(2.1)$ & $1.9(2.3)$ & $0.3(0.3)$ & $2.3(2.8)$ \\
$35 \mathrm{~km}$ & $12(11.2)$ & $11(10.2)$ & $6.1(5.7)$ & $0.2(0.1)$ & $1.4(1.3)$ & $2.3(2.1)$ & $0.5(0.5)$ & $1.5(1.4)$ \\
$30 \mathrm{~km}$ & $12(9.0)$ & $9.4(7.1)$ & $7.3(5.5)$ & $<0.1(<0.1)$ & $1.1(0.8)$ & $2.3(1.7)$ & $1.0(0.7)$ & $0.4(0.3)$ \\
$25 \mathrm{~km}$ & $13(8.0)$ & $9.0(5.6)$ & $9.1(5.6)$ & $0.2(0.1)$ & $3.2(2.0)$ & $1.2(0.7)$ & $0.6(0.3)$ & $0.8(0.5)$ \\
$20 \mathrm{~km}$ & $14(7.0)$ & $8.2(4.1)$ & $12(6.0)$ & $0.3(0.2)$ & $5.2(2.6)$ & $0.5(0.2)$ & $0.5(0.2)$ & $2.4(1.2)$ \\
$15 \mathrm{~km}$ & $13(5.6)$ & $7.1(3.1)$ & $11(4.7)$ & $0.3(0.1)$ & $1.2(0.5)$ & $0.8(0.3)$ & $1.6(0.7)$ & $2.8(1.2)$ \\
$10 \mathrm{~km}$ & $13(6.8)$ & $8.6(4.5)$ & $9.6(5.0)$ & $<0.1(<0.1)$ & $1.8(0.9)$ & $0.1(<0.1)$ & $2.1(1.1)$ & $2.0(1.0)$ \\
\hline
\end{tabular}

Table 2. Horizontal averaging kernels (full width at half maximum) calculated according to von Clarmann et al. (2009a) for retrieval altitudes between 40 and $10 \mathrm{~km}$ for HCFC-22. Positive sign means displacement towards the satellite.

\begin{tabular}{ccr}
\hline Height & FWHM & Displacement \\
\hline $40 \mathrm{~km}$ & $554 \mathrm{~km}$ & $-121 \mathrm{~km}$ \\
$35 \mathrm{~km}$ & $549 \mathrm{~km}$ & $5 \mathrm{~km}$ \\
$30 \mathrm{~km}$ & $528 \mathrm{~km}$ & $62 \mathrm{~km}$ \\
$25 \mathrm{~km}$ & $521 \mathrm{~km}$ & $55 \mathrm{~km}$ \\
$20 \mathrm{~km}$ & $608 \mathrm{~km}$ & $90 \mathrm{~km}$ \\
$15 \mathrm{~km}$ & $300 \mathrm{~km}$ & $133 \mathrm{~km}$ \\
$10 \mathrm{~km}$ & $327 \mathrm{~km}$ & $126 \mathrm{~km}$ \\
\hline
\end{tabular}

\section{Validation}

Validation of the HCFC-22 MIPAS IMK profiles is performed by comparison to coincident independent measurements. The availability of reference measurements for the validation of MIPAS HCFC-22 in the stratosphere is quite limited: the only space-borne instrument measuring the vertical profiles of HCFC-22 at the same time as MIPAS is ACE-FTS. We also perform the comparison with MkIV balloon profiles and with measurements at different heights performed with the balloon-borne cryosampler flown by the University of Frankfurt. Although aircraft measurements of HCFC-22 (e.g. Xiang et al., 2014) exist, they have not been used, due to unsolved problems caused by the different altitude resolutions of MIPAS and the air sampling measurements. The application of averaging kernels to homogenise profiles of different altitude resolution as suggested by Connor et al. (1994) is only possible if the better resolving instrument provides vertical profiles but not if values at a single altitude level are available only.

\subsection{Comparison with ACE-FTS}

The Atmospheric Chemistry Experiment Fourier Transform Spectrometer (ACE-FTS) is a solar occultation instrument flying on the SCISAT satellite platform since August 2003 (Bernath et al., 2005). It takes measurements from the upper troposphere to about $150 \mathrm{~km}$ altitude. Temperature, pressure, atmospheric aerosol extinction and the concentrations of a large number of atmospheric species are retrieved from these measurements with a vertical resolution on the order of $4 \mathrm{~km}$. The SCISAT flies on a highly inclined circular orbit $(650 \mathrm{~km}$ altitude), which implies that more than half of the ACE-FTS measurements occur at high (over $60^{\circ} \mathrm{N}$ and S) latitudes. It takes approximately 3 months for all latitudes $\left(\sim 82^{\circ} \mathrm{N}-82^{\circ} \mathrm{S}\right)$ to be sampled by ACE-FTS.

HCFC-22 is retrieved by the algorithm described in Boone et al. (2005) and Boone et al. (2013) from an analysis window of width $25 \mathrm{~cm}^{-1}$ centred at $817.5 \mathrm{~cm}^{-1}$. Between the tropopause and $30 \mathrm{~km}$ altitude a second, $27 \mathrm{~cm}^{-1}$ wide window is used, centred at $1114 \mathrm{~cm}^{-1}$. Information from the latter window dominates the retrieval. The ACE-FTS retrieved HCFC-22 profiles extend from the upper troposphere to about $30 \mathrm{~km}$ height, do not include any formal a priori information, and the reported errors are on the order of 3 to $5 \%$, going up to $10 \%$ at the lowest and $8 \%$ at the highest altitude limits of the retrieval. Spectroscopic data from HITRAN 2004 (Rothman et al., 2005) were used for HCFC-22 but for this species no updates have been made with respect to the HITRAN2K version used for MIPAS.

The analysis was performed on January 2005-April 2012 data of ACE-FTS version 3.5, with collocation criteria of $500 \mathrm{~km}$ and $5 \mathrm{~h}$; this leads to a comparison subset of 8393 collocated measurements. In the case of multiple matches, only the closest MIPAS profile was used.

The comparison of global mean MIPAS and ACE-FTS HCFC-22 profiles (left panel of Fig. 4) reveals a high MIPAS bias of 5 to $10 \mathrm{pptv}$ at $17-29 \mathrm{~km}$ altitudes and a low MIPAS bias (between 0 and $-3 \mathrm{pptv}$ ) at $10-13 \mathrm{~km}$. Between the altitudes of 13 and $22 \mathrm{~km}$, the mean difference is -3 to 

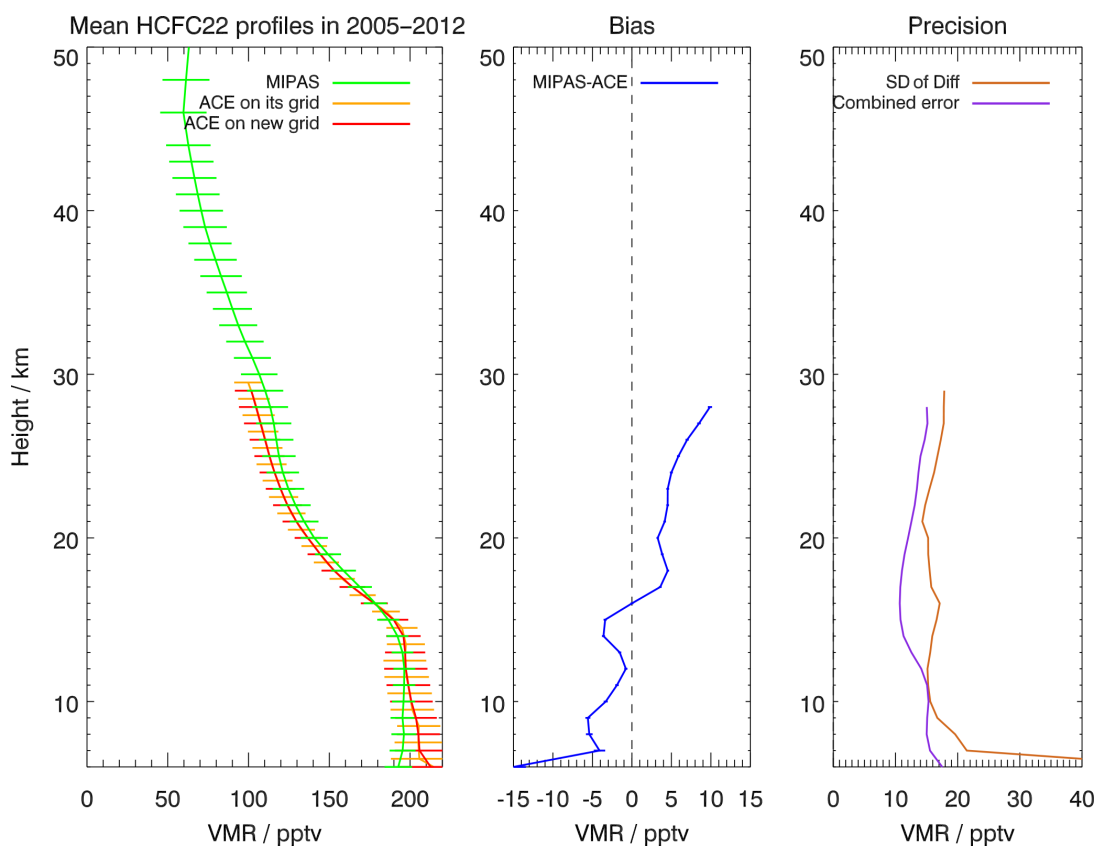

Figure 4. Mean profiles, bias and standard deviation of the differences vs. estimated combined retrieval error for ACE-FTS and MIPAS retrievals of HCFC-22. The original altitude grid on which the ACE-FTS data were provided uses the same grid spacing as MIPAS of 1-km, but is shifted by $0.5 \mathrm{~km}$. Thus, the data were resampled on the MIPAS grid. The error bars in the left panel are the typical errors of a single profile, estimated as the mean error over the sample. The tiny error bars in the middle panel (hardly discernable) are the standard errors of the mean differences.

+5 pptv. The bias is significant at all altitude levels. Analysis of the scatter of the differences vs. the estimated combined precision of the instruments (Fig. 4, right panel) indicates an underestimation of the combined uncertainty, i.e. one or both instruments underestimate the random component of their errors (cf. von Clarmann, 2006). It should, however, be kept in mind that the reported fitting error estimates of ACE-FTS include only measurement noise and do not include randomly varying parameter errors, which implies that perfect coincidence of the two curves on the right panel of Fig. 4 cannot be expected. Further, atmospheric variability within the radius defined by the coincidence criteria can contribute to these differences. This is particularly true because many of the ACEFTS measurements occur at higher northern latitudes where atmospheric variability is quite pronounced. Further, the use of different HCFC-22 bands may contribute to the observed differences.

The seasonality of the differences between ACE-FTS and MIPAS for southern polar latitudes is analysed in Fig. 5. Most pronounced differences occur at the top end of the ACE-FTS profiles, with ACE-FTS always lower than MIPAS, while the seasonality in the differences comes mainly from a very steep vertical gradient in the ACE-FTS profiles for polar summer between 16 and $20 \mathrm{~km}$ altitude (top left panel) which is not in the same way reproduced by MIPAS profiles. The very steep vertical gradient in this particular case leads to the highest bias of ACE-FTS vs. MIPAS of about $15 \mathrm{pptv}$ around $16 \mathrm{~km}$ and a low bias around $20 \mathrm{~km}$ and above.

The correlation plots of ACE-FTS vs. MIPAS (Fig. 6) corroborate the findings so far: below about $16 \mathrm{~km}$ altitude (red and yellow data points) the regression line is slightly steeper than unity indicating a bias proportional to the absolute values, while above $16 \mathrm{~km}$, ACE-FTS has a small and almost constant low bias vs. MIPAS.

The histogram plots (Fig. 7) represent the distribution of measured volume mixing ratios over latitude and time for a fixed altitude level. The histograms for ACE-FTS and MIPAS at $23 \mathrm{~km}$ are very similar in shape and position of the peak value, while at $16 \mathrm{~km}$ the ACE-FTS distribution of measured vmrs is somewhat wider. The latter is attributed to the fact that the ACE-FTS retrieval does not use any regularisation, which leads to greater scatter. Nevertheless, both peak and extreme values match quite well and the histograms suggest a robust agreement between the two instruments, albeit with an altitude-dependent systematic offset as discussed above. The bimodal distribution at $23 \mathrm{~km}$ altitude is caused by differences in the air masses sounded by each instrument, where the polar air masses correspond to the lower mode and the mid-latitude air masses to the higher mode. The number of tropical collocations is small and has thus minor impact on the histogram. The ACE-FTS modes are more clearly separated than the MIPAS modes because of the differences in the north-south component of the lines of sight of each 

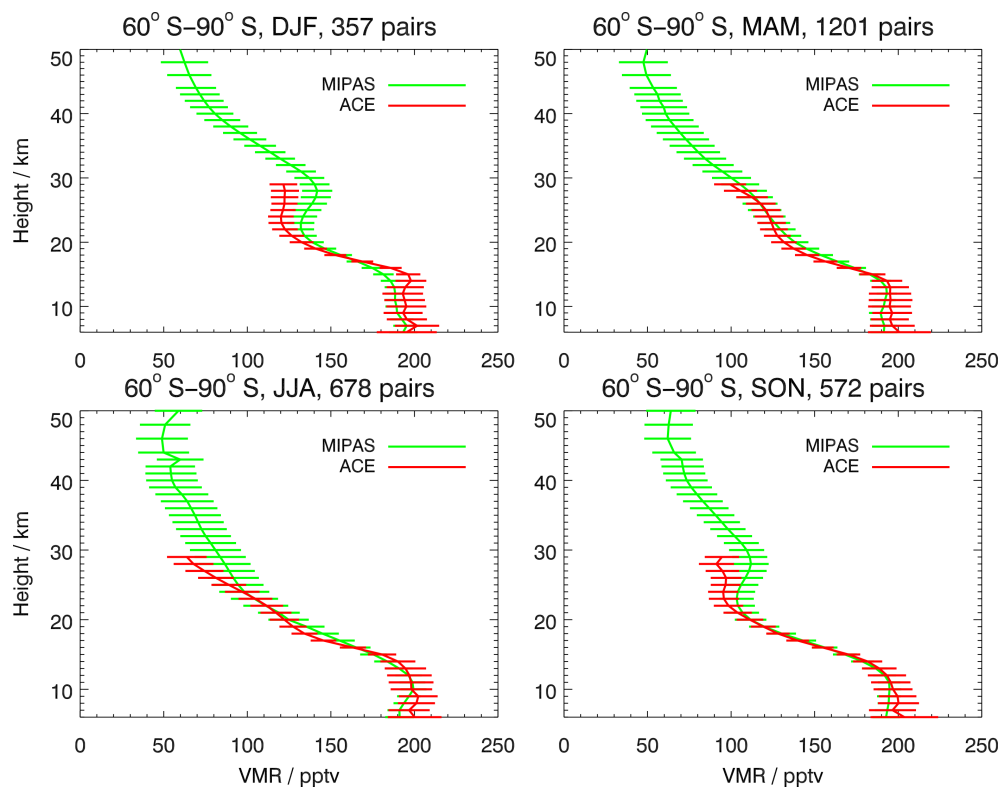

Figure 5. Seasonal mean vmr values (in pptv) of ACE-FTS (red) and MIPAS (green) in 2005-2012 at southern polar latitudes. Error bars represent the typical precision of a single profile, estimated as the mean error over the sample.

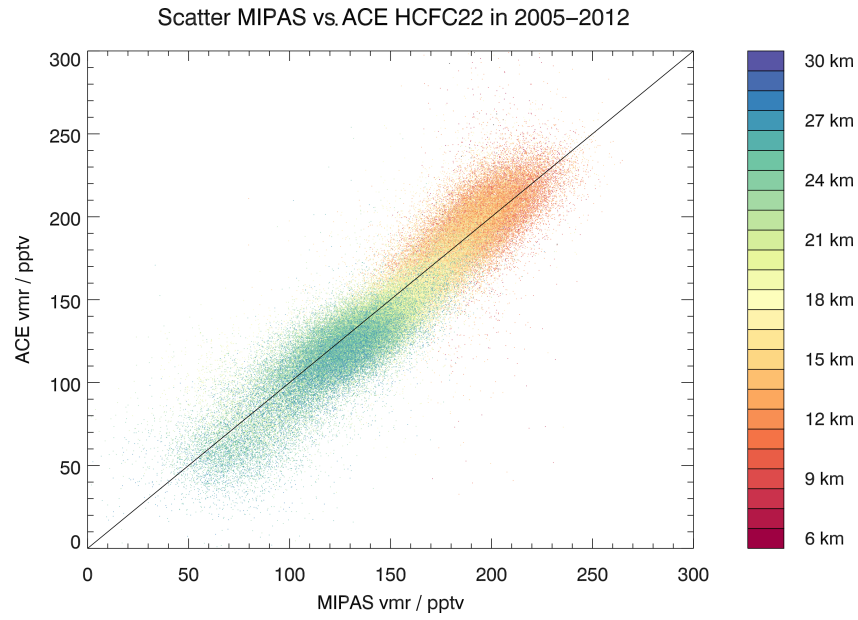

Figure 6. Scatter plot of ACE-FTS vs. MIPAS HCFC-22 data points.

measurement technique. Since the mixing ratio gradients in north-south direction are typically larger than those in eastwest direction, the modes of MIPAS that observes roughly in the orbit plane are more smeared than those of ACE-FTS whose line of sight is directed towards the sun.

In summary, although minor differences between MIPAS and ACE-FTS HCFC-22 measurements have been detected, the comparison justifies confidence in the data sets. Besides identified small biases on the order of $<10$ pptv the two data sets compare very well in absolute values, latitude distributions, and seasonalities.

\subsection{Comparison with cryosampler profiles}

The cryogenic whole air sampler, deployed on stratospheric balloons, is operated by the University of Frankfurt. The instrument collects high volume whole air samples which are frozen out by means of liquid neon. After the flight, the air is left to evaporate which provides high pressure whole air samples from different altitudes (Engel et al., 1997). A wide range of halocarbons are then analysed in these samples using a gas chromatograph coupled to a mass-spectrometer (Laube et al., 2008). The precision of the individual data points of the cryosampler measurements is typically on the order of $0.5 \%$. The measurements were referenced to a standard provided by the National Oceanic and Atmospheric Administration (NOAA) (e.g. Montzka et al., 2003) and the data were reported on the NOAA-2006 calibration scale, which shows excellent agreement (within 1\%) to most other calibration scales (Hall et al., 2014; WMO, 2014).

Cryosampler measurements do not represent contiguous vertical profiles but rather individual independent point measurements. Hence, no regridding has been applied in this case: the cryosampler measurements were just reported as they were on the height where they had been taken.

Coincidences between MIPAS and cryosampler measurements were considered within a spatial distance of $1000 \mathrm{~km}$ and a time window around the cryosampler measurement time of $\pm 24 \mathrm{~h}$. These coincidence criteria may seem large. However, in their work on the validation of CFC-11/CFC12 and $\mathrm{CH}_{4}$ measurements from MIPAS, where data from the same MIPAS geolocations and balloon flights were used, Eckert et al. (2015) and Laeng et al. (2015) found remark- 

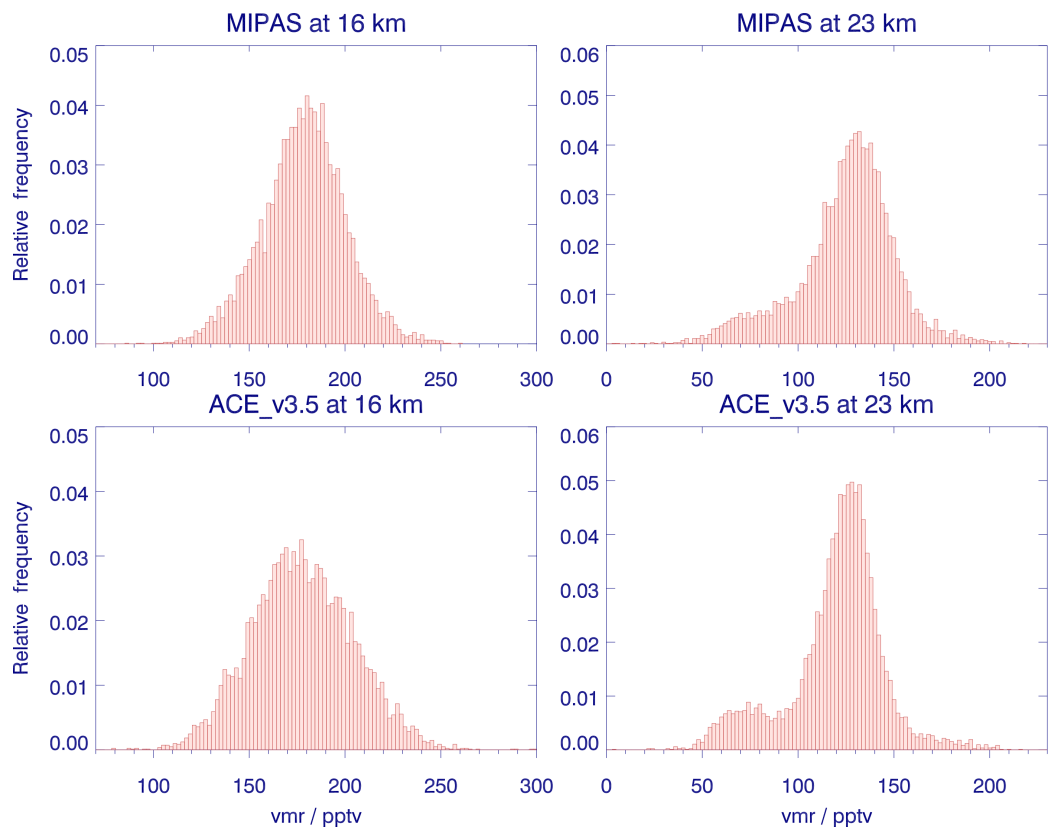

Figure 7. Histograms of MIPAS (upper panels) vs. ACE-FTS (lower panels) HCFC-22 mixing ratios at $16 \mathrm{~km}$ (left panels) and $23 \mathrm{~km}$ (right panels) altitude.

ably good agreement between MIPAS and the cryosampler data, except for biases confirmed by other validation instruments. Thus, it is rather unlikely that any differences found in the HCFC-22 comparison can be attributed to the relatively large spatial or temporal distance.

For the first two flights (first two panels of Fig. 8) that took place in June 2005 in the tropics the agreement between MIPAS profiles and cryosampler measurements above $30 \mathrm{~km}$ altitude is better than 10 pptv. Nevertheless, the MIPAS profile with the closest coincidence differs significantly from the other coincident profiles in both cases, hinting towards inhomogeneous situations in the atmosphere. Below $30 \mathrm{~km}$, for the first flight neither the closest coincident profile, nor the mean of coincidences agrees with the cryosampler measurements. MIPAS data are higher than the cryosampler measurements between 15 pptv (mean coincident profile) and $35 \mathrm{pptv}$ (closest profile). For the second flight, the cryosampler data points fall mostly within the error bars of the closest MIPAS profile.

In order to eliminate the effect varying dynamical conditions within the quite large coincidence criterion, the HCFC22 profiles are also shown using the CFC- 12 measurements of the respective instrument as altitude coordinate in a similar way as done by von Clarmann et al. (1995). Since both species are subject to the same vertical transport, profile differences surviving this transformation can no longer be attributed to different meteorological conditions of the air parcels measured, and thus are considered significant. CFC-12 was chosen as a transfer standard between MIPAS and the cryosampler data because this species is available from both instruments and because MIPAS CFC-12 measurements were found to be the most reliable of all MIPAS measurements of long-lived gases (Eckert et al., 2015; Laeng et al., 2015). This representation confirms the findings discussed above, namely that MIPAS mixing ratios are higher than those of the cryosampler at tropospheric altitudes but agree reasonably well above (blue and red curves/symbols in Fig. 9).

The third flight (third panel of Fig. 8) of the cryosampler instrument provided only four measurements, none of which being situated between 18 and $32 \mathrm{~km}$. For all four data points, MIPAS is higher by $15-30$ pptv. This finding is confirmed using CFC-12 as a transfer standard (green curves/symbols in Fig. 9).

The cryosampler data of the fourth flight (fourth panel) show a narrow layer with very low HCFC-22 abundances around $23 \mathrm{~km}$; within this layer the abundances are mostly far lower than the closest MIPAS profile. Pronounced oscillations appear also in some but not all individual MIPAS profiles, but have, where present, a significant high bias (20 to 50 pptv) compared to the cryosampler data. Other individual MIPAS profiles, in particular the profile of the closest coincidence, are rather smooth. The oscillating cryosampler data are attributed to an unusual atmospheric situation on this particular flight with a narrow lamina of HCFC-22 poor polar vortex air, and this situation led to strong small-scale variability. This hypothesis is corroborated by the large spread of the collocated MIPAS profiles. The lamina itself is either not encountered by the MIPAS measurements, or it is not resolved; MIPAS measurements represent for each pro- 
file point an air parcel of about $400 \mathrm{~km}$ in length times $30 \mathrm{~km}$ in width times $4-9 \mathrm{~km}$ in height. Below $18 \mathrm{~km}$, the MIPAS profiles have a high bias of 15 to 20 pptv. The representation based on CFC-12 as a transfer standard (pink curve/symbols in Fig. 9) confirm the good agreement between both instruments except for the one data point where the cryosampler data show a pronounced minimum.

The last flight (bottom panel of Fig. 8) stands out by a pronounced HCFC-22 minimum (also present in the profiles of other tracers measured during this flight) in MIPAS data at approximately $28 \mathrm{~km}$. Below this altitude, the cryosampler measurements coincide reasonably well with the MIPAS data but the altitude coverage of the cryosampler data set on this day does not allow to confirm the positive mixing ratio gradient above. Except for the lowermost datapoints, the agreement between the MIPAS and the cryosampler measurements is excellent in the representation using CFC-12 as transfer standard (brown curve in Fig. 9).

In summary, comparison to cryosampler data from five different profiles from tropical and northern polar winter atmosphere reveal a high bias of MIPAS HCFC-22 data of 15 to 50 pptv below $24 \mathrm{~km}$; while above $28 \mathrm{~km}$, the high bias is reduced to less than 10 pptv.

\subsection{Comparison with MkIV balloon interferometer profiles}

The MkIV interferometer from Jet Propulsion Laboratory (JPL) is a high-resolution solar absorption spectrometer for measurement of over 30 atmospheric constituents which is deployed on stratospheric balloon platforms with a typical float altitude of $37 \mathrm{~km}$ (Toon, 1991). MkIV measured HCFC22 using two spectral windows, centred at the $v_{4}$ Q-branch at $809.19 \mathrm{~cm}^{-1}$ and the $2 v_{6}$ Q-branch at $829.14 \mathrm{~cm}^{-1}$.The widths of the microwindows were 1.28 and $0.72 \mathrm{~cm}^{-1}$, respectively. Pseudolines derived from spectroscopic measurements (McDaniel et al., 1991; Varanasi, 1992; Varanasi et al., 1994) have been used as spectroscopic data (see http:// mark4sun.jpl.nasa.gov/pseudo.html). The instrument obtains vmr vertical profiles of HCFC-22 between cloud top and balloon altitude. MkIV measured two HCFC-22 vmr vertical profiles collocated by MIPAS reduced resolution measurements. The data set is provided on a $1-\mathrm{km}$ altitude grid between 10 and $40 \mathrm{~km}$. The vertical resolution of the MkIV balloon profiles varies between 2-4 km. Data used here were measured during balloon flights from Fort Sumner, NM, with tangent altitude geolocations from $34.0-35.7^{\circ} \mathrm{N}$ and 108.8 $114.1^{\circ} \mathrm{W}$.

Figure 10 presents the two MkIV balloon profiles within the MIPAS reduced resolution period. The first MkIV profile, from 22 September 2007, was measured when MIPAS was temporarily inactive and no matches were found within $24 \mathrm{~h}$ and $1000 \mathrm{~km}$. The MkIV profile from 22 September 2007 was compared to the MIPAS $30-40^{\circ} \mathrm{N}$ monthly mean of September 2007. These observations were made at the end
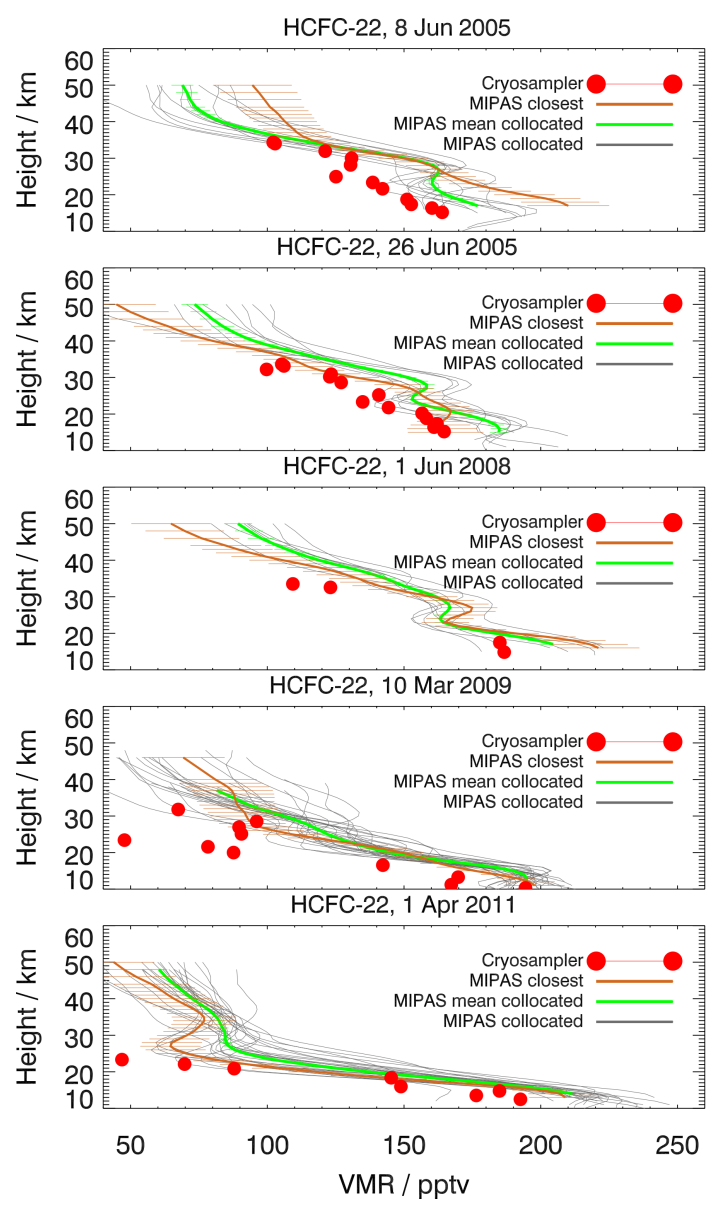

Figure 8. Five cryosampler profiles (red filled circles) and MIPAS HCFC-22 vmr profiles - all collocated (grey), closest collocations (orange) and the mean profiles from all collocations (green).

of a summer of easterly stratospheric winds. Since the flow is zonal during the summer and wave activity is negligible, little zonal variation in composition is to be expected, which justifies comparison with a zonal monthly mean of the same year. For the profile from the sunrise of 23 September 2007, three collocated MIPAS profiles were found (grey lines).

Below $25 \mathrm{~km}$ the MIPAS profiles agree with the MkIV profiles within the error bars, with MIPAS HCFC-22 being in tendency lower for the collocated profiles, (right panel) but in good agreement for the September 2007 monthly mean. Above $25 \mathrm{~km}$ the vertical gradients of mixing ratios from MkIV and MIPAS diverge, with MkIV profiles decreasing stronger with altitude than those of MIPAS. Up to $32 \mathrm{~km}$, the agreement is still well within the error bars. For the 22 September 2007 comparison the profiles agree even up to the highest MkIV altitude levels.

\subsection{Summary of the intercomparisons}

The comparisons to the three available reference data sets, namely ACE-FTS, balloon-borne cryosampler data and 


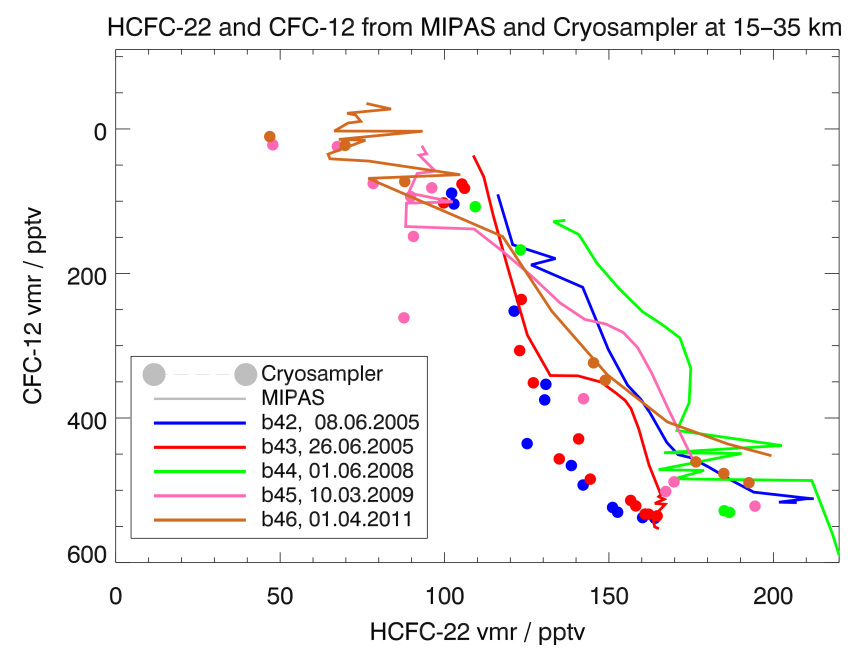

Figure 9. Comparison of the five cryosampler HCFC-22 profiles (dots) with the closest MIPAS profiles (continuous lines) using the respective CFC-12 data as altitude coordinate. In this representation differences caused by different meteorological conditions are eliminated.

MkIV balloon measurements, do not provide a unique picture on MIPAS biases. Below about $20 \mathrm{~km}$, MIPAS has either a low (ACE-FTS), a high (cryosampler) or no (MkIV) bias. Above $25 \mathrm{~km}$ the MIPAS bias is either clearly positive (ACE-FTS and MkIV) or small, i.e. less than +10 pptv (cryosampler). Between 20 and $25 \mathrm{~km}$ the bias can range from $-30 \mathrm{pptv}$ (MkIV) to $+50 \mathrm{pptv}$ (cryosampler). In summary we state that there is no clear indication of a bias, and MIPAS does not stand out as particularly high or low. MIPAS HCFC-22 data are found to be within \pm 15 pptv $(1 \sigma)$ of reference data sets between 10 and $35 \mathrm{~km}$ altitude. The different size of the reference data sets needs to be taken into account. The large number of collocations with ACE-FTS leads to a high statistical significance of deviations with respect to noise. Errors of systematic nature, however, do not cancel out by averaging, and with respect to these, the balloon measurements are considered equally useful despite the small number of collocations. In addition, cryosampler data do not rely on spectroscopic measurements as the other three data sets and, thus, are the most independent reference data within the intercomparisons. Disagreement with cryosampler data hints towards a potential bias of all spectral measurements due to incorrect spectroscopic information.

\section{Global distributions and temporal evolution}

\subsection{Zonal means}

Figures 11 and 12 show monthly zonal means of HCFC$22 \mathrm{vmr}$ for December 2005 and 2010 and for July 2006 and 2010, respectively, for all latitudes and altitudes up to $50 \mathrm{~km}$. The months have been selected to be approximately in the
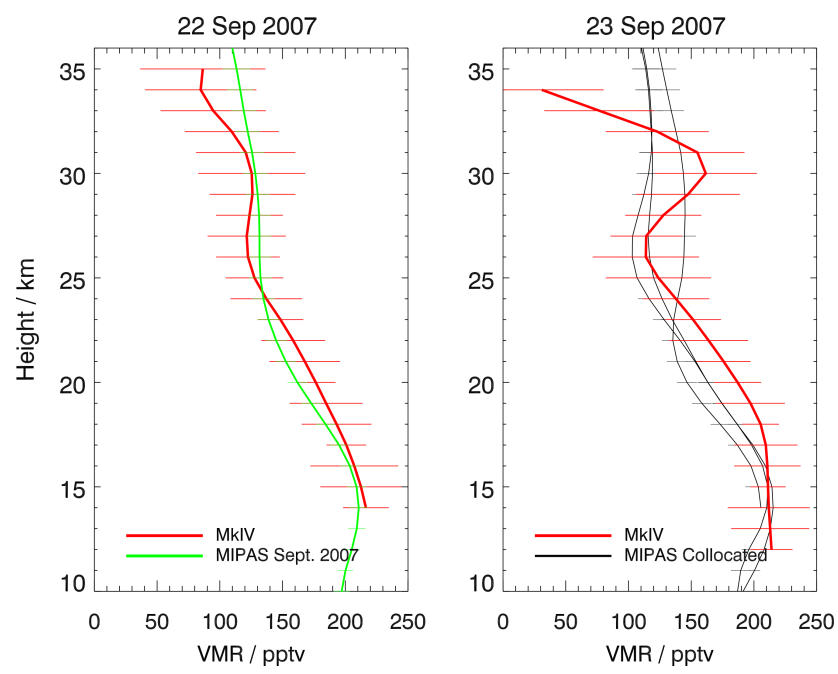

Figure 10. MkIV and MIPAS collocated and monthly mean vertical HCFC-22 profiles along with error bars. The mean profile is taken in the $30-40^{\circ} \mathrm{N}$ latitude band where the two balloon flights took place.

same phase of the quasi-biennial oscillation (QBO). The typical distribution of a tropospheric source gas with photolytic sinks in the stratosphere is observed, with higher values in the tropics and lower altitudes, and lower values in the upper stratosphere and higher latitudes. For austral polar summer (Fig. 11), a relative HCFC-22 maximum is seen between 20 and $30 \mathrm{~km}$. This maximum is a remnant of the vortex breakdown. As already demonstrated in Stiller et al. (2012), mid-latitude (i.e. HCFC-22-richer and younger) air is transported towards the South Pole at the time of the vortex breakdown and separates the vortex into an upper and a lower part. This situation is seen in Fig. 11. After the intrusion, the midlatitude air mixes with the lower part of the vortex air.

Within the troposphere, MIPAS sees larger HCFC-22 abundances in the Northern Hemisphere (NH) than in the Southern Hemisphere (SH), owing to the global distribution of emissions, which is in agreement with previous results from Xiang et al. (2014), while in the stratosphere this kind of asymmetry is not observed. Furthermore, a substantial increase of HCFC-22 from the years 2005-2006 to the year 2010 can be derived from the MIPAS results.

The HCFC-22 distributions show a maximum in the tropical upper troposphere which is, at first glance, not expected for a source gas emitted at the ground. Closer inspection, however, reveals that in 2-D distributions, the situation is different, if we assume that there are extratropical localised sources and a localised uplift region and, after a certain altitude has been reached, injection into the zonal transport direction of the tropics. A source gas maximum as observed by MIPAS then can be explained by the following mechanism: due to the local uplift of polluted air from localised sources, the enhancement of the pollutant averages out to a certain de- 

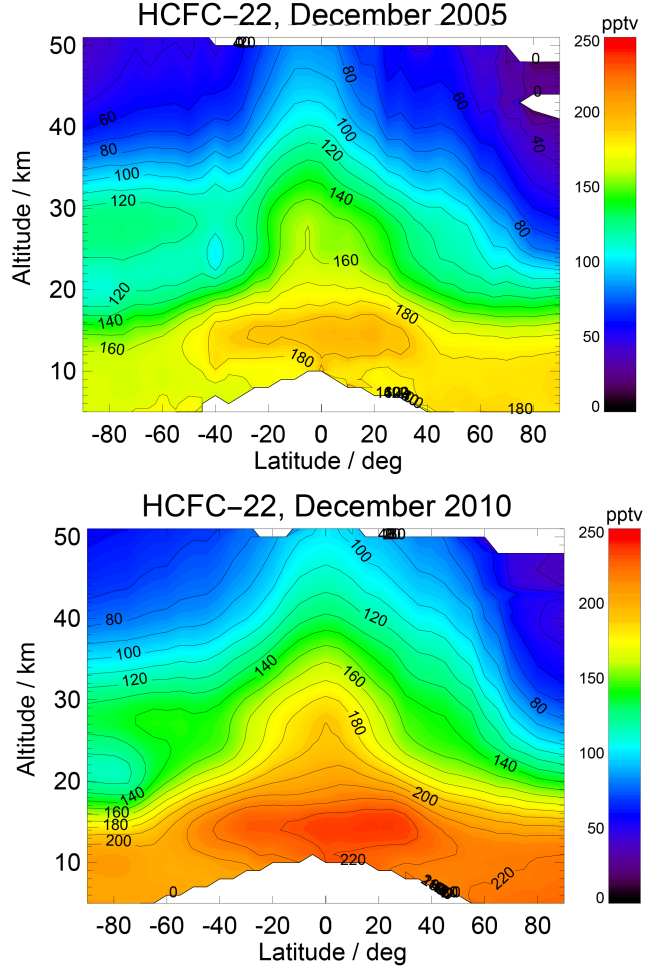

Figure 11. Monthly zonal means of HCFC-22 volume mixing ratios from 5 to $50 \mathrm{~km}$ for December 2005 (top) and December 2010 (bottom) as a function of geometrical altitude. The months have been selected to be approximately in the same QBO phase.

gree when the zonal mean values for the lower altitudes are calculated. At higher altitudes the transport direction turns to zonal and averaging happens along the transport direction, involving summation over a series of enhanced values, and the reduction of the zonal mean by averaging over polluted and clean air masses no longer takes place.

In the case of MIPAS HCFC-22, highest vmrs of up to $240 \mathrm{pptv}$ are observed at the end of the observation period, and occur at altitudes between 10 and $15 \mathrm{~km}$ at $30-50^{\circ} \mathrm{N}$ during boreal summer and coincide with the position of the Asian monsoon anticyclone (Fig. 13, upper panel). After the break-down of the Asian monsoon anticyclone, during boreal fall, these HCFC-22 enhancements are spread over all longitudes and transported into the TTL (Tropical Tropopause Layer), where they are subsequently distributed over the tropics, providing the isolated maximum layer of up to $225 \mathrm{pptv}$ at low latitudes (Fig. 13, middle panel). This behaviour is consistent with the general explanation scheme outlined above: HCFC-22 production was not restricted for developing countries during the relevant time, and some of these countries, e.g. China, have been large HCFC producers for years, while HCFC-22 production is controlled by the Montreal Protocol for industrialised countries. Indeed, Saikawa et al. (2012) found a surge in HCFC-22 emissions between 2005 and 2009 from developing countries in Asia
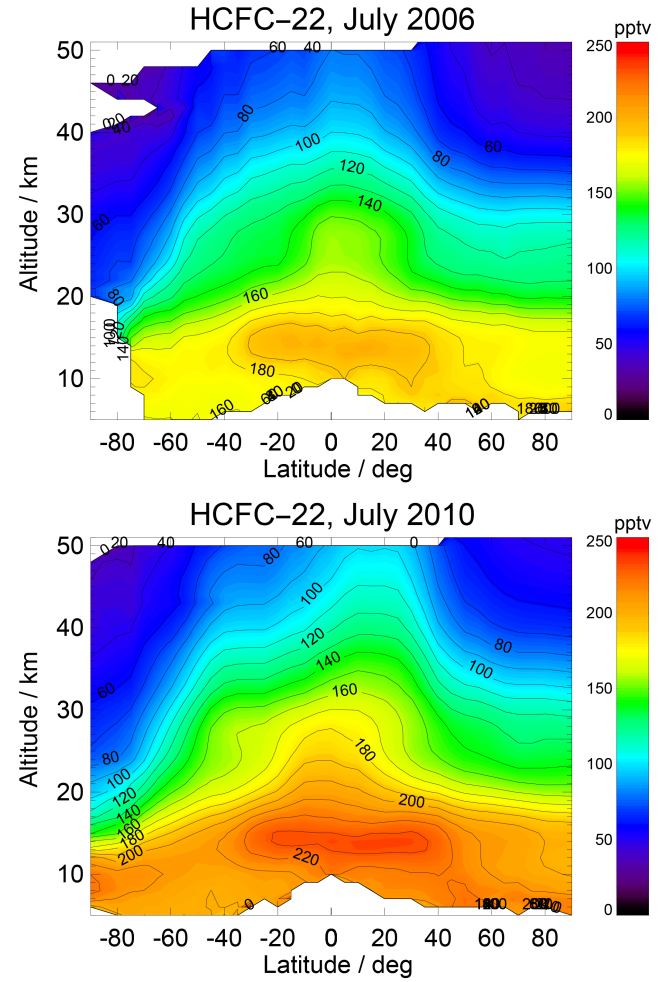

Figure 12. Same as Fig. 11, but for July 2006 (top) and 2010 (bottom).

with the largest emitting region including China and India. Also Montzka et al. (2009) suggested a shift from high to low latitude emissions during this period, consistent with these assertions. High HCFC-22 abundances from these industrial regions of Asian developing countries are transported upwards into the Asian monsoon anticyclone during summer. Transport calculations based on meteorological analyses confirm that emissions from these regions indeed feed the Asian monsoon system (Vogel et al., 2015). After having been lifted into close-to-tropopause levels inside the Asian monsoon anticyclone, the high HCFC-22 abundances are transported into the tropical tropopause region (Fig. 13, lower panel). Transport from the Asian monsoon anticyclone into the tropical tropopause layer as a dominant source of tropical seasonality was suggested by Ploeger et al. (2012) based on model experiments, and by Randel and Jensen (2013). Once intruded into the TTL, the enhanced HCFC-22 abundances are distributed over all the tropical longitudes and generate an isolated maximum layer between appr. $10 \mathrm{~km}$ and the tropopause. This process also may explain why the isolated HCFC-22 vmr maximum observed by MIPAS above about $10 \mathrm{~km}$ exceeds abundances measured at the surface at remote sites in mid-latitudes by both the Global Monitoring Division of NOAA's Earth System Research Laboratory (NOAA/GMD) and the Advanced Global Atmospheric Gases Experiment (AGAGE) (cf. Sect. 5.4). 

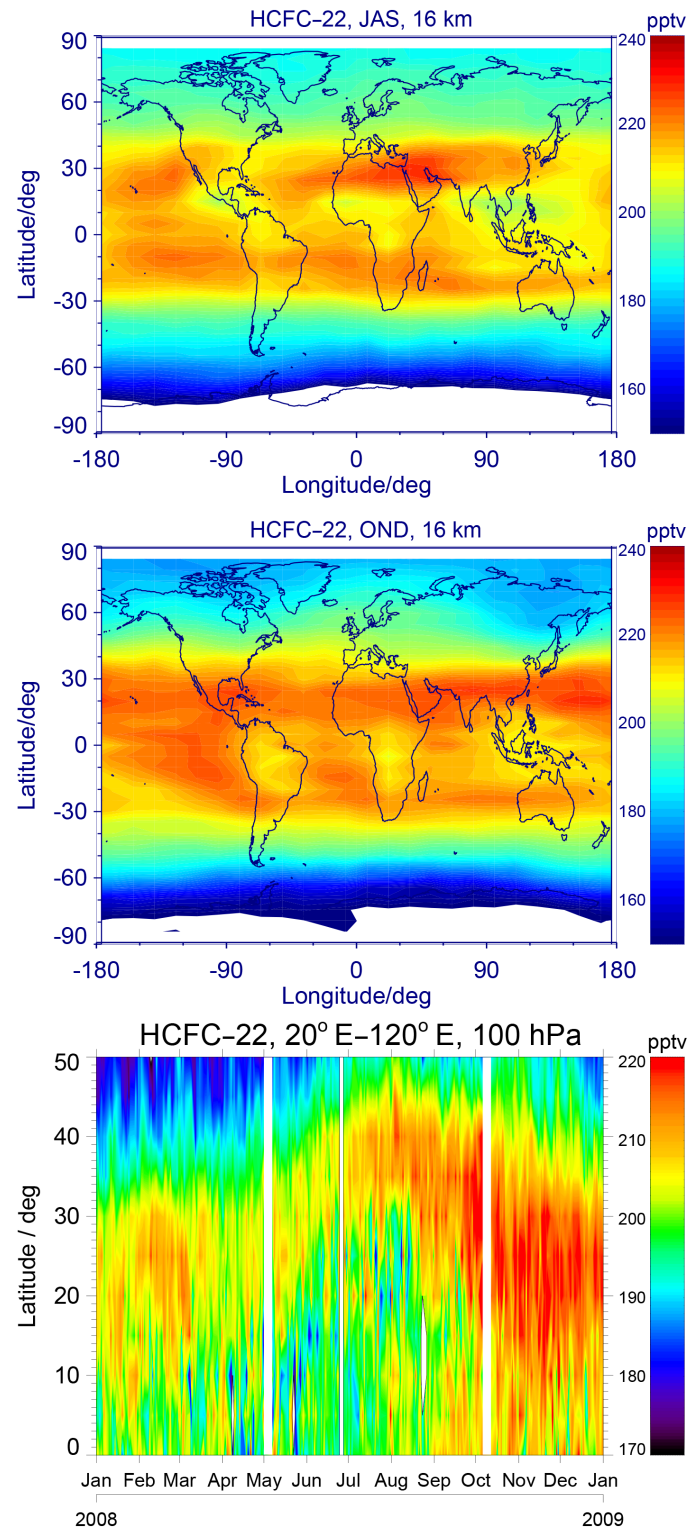

Figure 13. (top) Global HCFC-22 distribution in July-AugustSeptember for the years 2005 to 2011 at $16 \mathrm{~km}$ altitude. An enhancement over the Asian monsoon region is clearly visible. (middle) Global HCFC-22 distribution in October-NovemberDecember for the years 2005 to 2011 at $16 \mathrm{~km}$ altitude. The maximum previously located over the monsoon region has spread over all longitudes now. (bottom) The temporal development of HCFC22 in the Asian monsoon region (i.e. daily averaged over 20 to $120^{\circ} \mathrm{E}$ and shown for 0 to $50^{\circ} \mathrm{N}$ in $5^{\circ}$ bins) at $100 \mathrm{hPa}$ for the year 2008. Enhanced values are seen to propagate towards the tropics from August on.

\subsection{Time series analysis of HCFC-22 for various altitudes and latitudes}

The MIPAS HCFC-22 data presented here cover the socalled reduced resolution phase from January 2005 to

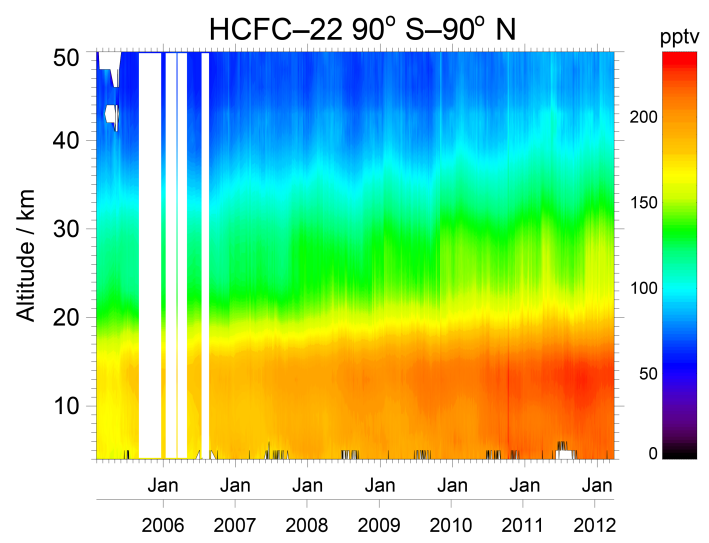

Figure 14. The globally averaged time series of HCFC-22 volume mixing ratio from January 2005 to April 2012.

April 2012. The instrument did not measure continuously in the nominal mode. This leads to frequent data gaps, but they are relatively short on average ( 1 to 2 days, occasionally longer). Some days were filtered out as well, since they contain too few measurements, and for certain phases there are data gaps in certain latitudes due to calibration measurements always performed at the same latitudes.

The averaged global time series of HCFC-22 volume mixing ratio from January 2005 to April 2012, including all latitudes from the South to the North Pole is shown in Fig. 14. The global mean HCFC-22 volume mixing ratio increases at all altitudes with time. The global mean HCFC22 volume mixing ratio at, for example, $16 \mathrm{~km}$ altitude was about 161 pptv in January 2005, and it increased up to about 210 pptv by April 2012. This provides us with a rough estimate of the increase of HCFC-22 content: it had increased by 49 pptv in 7 years, which is roughly 7 pptv per year.

For a more detailed analysis, we consider the mean mixing ratios in $20^{\circ}$ latitude bands (Fig. 15). In this figure, it is again visible that the zonal mean abundance of HCFC-22 over the equator is much higher than over the poles at similar altitudes. In addition to the latitude dependence of the absolute volume mixing ratios, the oscillations due to the seasonal cycle are more pronounced at higher latitudes for the stratosphere, while a pronounced seasonal cycle in the upper troposphere can also be found at $30-50^{\circ} \mathrm{N}$. In the stratosphere at the poles, any displacement of the polar vortex from the poles or its deformation leads, in a 2-D representation, to sharp vortex boundaries being smeared out by zonal averaging. In contrast, a breakup of the polar vortex would finally lead to physical mixing. In a 2-D representation, physical mixing and zonal averaging effects cannot be distinguished in any obvious way, but both processes would explain a rapid increase of stratospheric zonal mean polar HCFC-22 mixing ratios. Indeed, the onset of polar vortex displacement and distortion due to wave activity has been verified to coincide in time with the observed increase of polar stratospheric HCFC- 

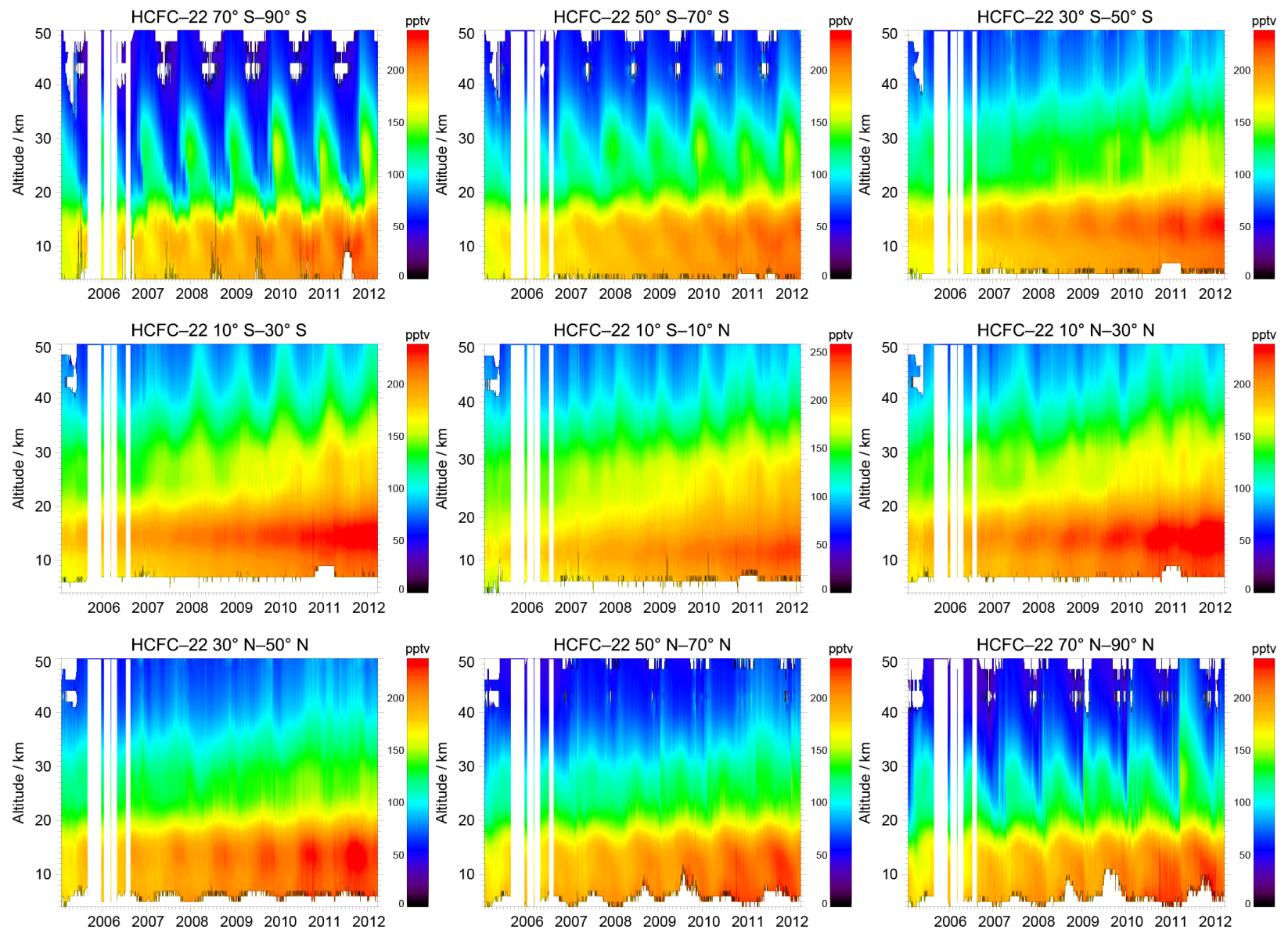

Figure 15. Time series of HCFC-22 vmrs from January 2005 to April 2012 for latitude bands (top row from left to right: 70 to $90^{\circ} \mathrm{S}, 50$ to $70^{\circ} \mathrm{S}$ and 30 to $50^{\circ} \mathrm{S}$; middle row from left to right: 10 to $30^{\circ} \mathrm{S}, 10^{\circ} \mathrm{S}$ to $10^{\circ} \mathrm{N}$ and 10 to $30^{\circ} \mathrm{N}$; bottom row from left to right: 30 to $50^{\circ} \mathrm{N}$, 50 to $70^{\circ} \mathrm{N}$ and 70 to $90^{\circ} \mathrm{N}$ ). The time series were generated from daily zonal means. White areas in the plots represent MIPAS data gaps. At low altitudes these are caused by clouds, while at high altitudes they are caused by a too low sensitivity of MIPAS. Data gaps covering all altitudes are associated with times when MIPAS did not measure.

22 abundances. Interestingly, the sudden increase of polar stratospheric HCFC-22 seems to take place at all altitudes at almost the same time for the northern polar region, while for the southern polar region the increase of HCFC-22 is observed to start at around $30 \mathrm{~km}$ and to slowly move down. Thus low HCFC-22 abundances are observed to last longer at lower altitudes. This is explained by the fact that the increase at the northern latitudes is caused by wave activity along with zonal averaging effects as discussed above, while in the Southern Hemisphere it is caused by the breakdown of the polar vortex. The first kind of processes happens almost at the same time at all altitudes, while the vortex break-down is known to start at higher altitudes and to proceed downwards.

In this context it is also interesting to see that a local maximum of HCFC-22 appears just after austral vortex break-up around $30 \mathrm{~km}$ altitude (most pronounced in the years 2009 to 2011), which indicates that young HCFC-22-rich air from low latitudes is rapidly transported into the polar region at these altitudes. A similar observation has been made within the analysis of global distributions of mean age of stratospheric air (AoA) (Stiller et al., 2012).

In mid-latitudes in the middle and upper stratosphere, there is no clear seasonal cycle visible, while at lower altitudes, in the lower stratosphere (below $20 \mathrm{~km}$ ), the maxima are shifted towards hemispheric late summer/fall. This is consistent with the current picture of the seasonal cycle of the Brewer-Dobson circulation, where the extra-tropical lower stratosphere is thought to be flooded by young tropical air during summer when the subtropical jet forms a weak mixing barrier only (Bönisch et al., 2011; Birner and Bönisch, 2011; Stiller et al., 2012).

Figure 16 provides the time series of HCFC-22 as latitudetime cross sections for certain altitude levels. At 10 and $14 \mathrm{~km}$, high seasonality in the northern subtropical latitudes with maxima during summer and caused by the Asian monsoon uplift is obvious. Around $20 \mathrm{~km}$ a clear seasonal cycle at high latitudes can be seen. Around $30 \mathrm{~km}$, a QBO variation in 

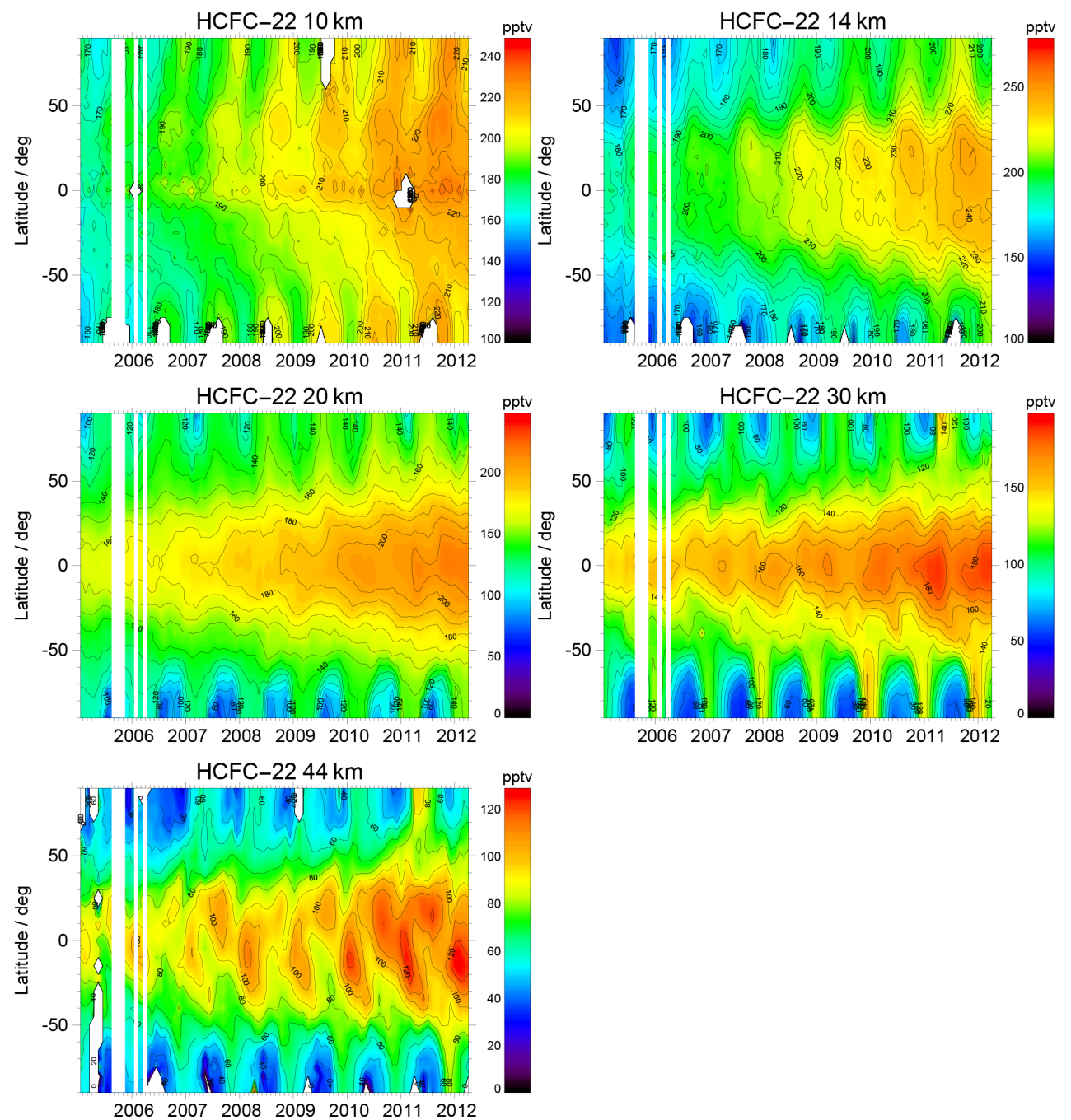

2006200720082009201020112012

Figure 16. Latitude-time cross sections of HCFC-22 vmrs from January 2005 to April 2012 for altitudes of 10, 14, 20, 30 and 44 km (from top left, top right, etc. to bottom left), generated from monthly zonal mean data. Note different colour bars for different panels.

the tropics modulates the seasonal variation, while at $44 \mathrm{~km}$ the pattern is dominated by the semi-annual oscillation.

Revisiting Fig. 14, it is obvious that the globally averaged distribution of HCFC-22 vmr in the stratosphere reveals also seasonal oscillations. While one might expect that $\mathrm{NH}$ and SH seasonal cycles average perfectly out on a global scale, this seems not to be the case. This is due to the fact that the SH seasonal cycle is not quite in the opposite phase to the cycle in the NH (see Fig. 16). In addition, the HCFC22 seasonal cycle in the $\mathrm{SH}$ is more pronounced than in the $\mathrm{NH}$, which is attributed to the more stable polar vortex which dominates the SH seasonal cycle. Hence, the seasonal cycle in the SH has a higher amplitude and thus causes a residual seasonal cycle on a global scale. The hemispheric tropospheric means of HCFC-22 vmr (Fig. 16, top left panel) for the $\mathrm{NH}$ are generally higher than for the $\mathrm{SH}$. This is due to higher industrial production of HCFC-22 in the NH.

\subsection{Trend of HCFC-22}

We have analysed time series of monthly means at specific altitudes for $10^{\circ}$-wide latitude bands by fitting the following regression function to the data:

$$
\begin{aligned}
\operatorname{vmr}(t) & =a+b t+c_{1} \mathrm{qbo}_{1}(t)+d_{1} \mathrm{qbo}_{2}(t) \\
& +\sum_{n=2}^{9}\left(c_{n} \sin \frac{2 \pi t}{l_{n}}+d_{n} \cos \frac{2 \pi t}{l_{n}}\right),
\end{aligned}
$$

where $t$ is time, $a$ is the axis intercept, and $b$ represents the linear component of the temporal variation, which for reasons of simplicity we call "trend", without claiming that it has any climatological meaning beyond the time window under investigation. $\mathrm{qbo}_{1}$ and $\mathrm{qbo}_{2}$ are $\mathrm{QBO}$ indices, and the terms under the sum are 8 sinusoidal functions of the period length $l_{n}$. The terms $\mathrm{qbo}_{1}$ and $\mathrm{qbo}_{2}$ are the normalised Singapore winds at 30 and $50 \mathrm{hPa}$ as provided by the Free 

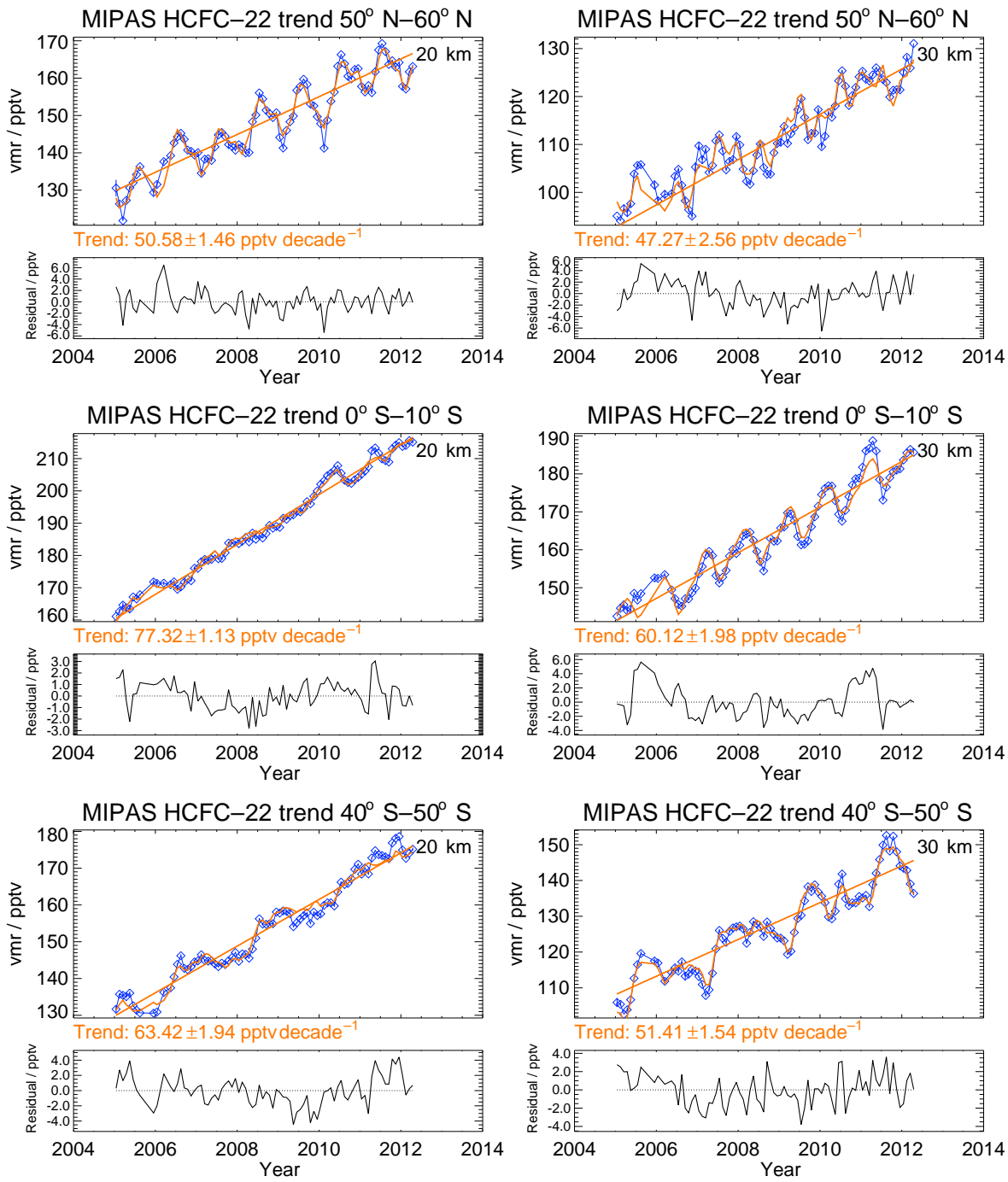

Figure 17. Time series of HCFC-22 volume mixing ratios (blue symbols) and their fits with the regression function described in the text (orange curves) at $20 \mathrm{~km}$ (left) and $30 \mathrm{~km}$ (right) altitude in three latitude bins. The linear trend component of the multi-parameter fit (straight orange line) is also shown.

University of Berlin via http://www.geo.fu-berlin.de/met/ag/ strat/produkte/qbo/index.html. qbo 1 and $\mathrm{qbo}_{2}$ are approximately orthogonal such that their combination can emulate any QBO phase shift (Kyrölä et al., 2010). Coefficients $a, b$, $c_{1}, \ldots, c_{9}, d_{1}, \ldots, d_{9}$ are fitted to the data using the method by von Clarmann et al. (2010), where the full error covariance matrix of the HCFC-22 data is considered, with the squared standard errors of the mean (SEM) of the monthly zonal means as the diagonal terms. Further, a constant model uncertainty error term has been added to the data error covariance matrix, which represents the deficiencies of the regression model with respect to the true atmospheric variation and was, within an iterative procedure, scaled such that the resulting $\chi_{\text {reduced }}^{2}$ of the trend fit was close to unity, corresponding to combined data and model uncertainties consistent to the fit residuals. Since we cannot exclude that these perturba- tions to be accounted for by this additional error term have a typical duration of more than 1 month, covariance terms between adjacent data points were also considered in order to account for the resulting autocorrelation. Phase shifts of the variations are represented by common use of sine and cosine functions of the same period length. The first and the second sinusoidal functions represent the seasonal and the semi-annual cycles, and have the periods of 12 and 6 months, respectively. To model the deviations of the temporal variation from pure sine or cosine shapes, i.e. to allow for irregular shapes like sawtooth shapes etc., the period lengths of the remaining six terms under the sum are chosen to be equal to $3,4,8,9,18$ and 24 months. The general strategy of this particular fitting has been described in Stiller et al. (2012).

Figure 17 provides some examples for time series and their fits for 3 different latitude bins and two altitudes. (40 to $50^{\circ} \mathrm{S}$, 
0 to $10^{\circ} \mathrm{S}$ and 50 to $60^{\circ} \mathrm{N}$, at 20 and $30 \mathrm{~km}$, respectively). The lower panel of each figure provides the residuals between measured and fitted time series. The simple model is able to represent the observations very well in most cases. Besides the linear increase, for all altitude/latitude bins, a more or less pronounced seasonal cycle is the dominant feature of the time series. In the southern mid-latitudes, a clear QBO signal is also present. For the northern mid-latitudes at $30 \mathrm{~km}$, we see also the impact of the semi-annual variation. The highest amplitudes in the temporal variation are reached in the $60^{\circ}$ regions; in the inner tropics, the amplitudes are lower, particularly in the lower stratosphere. A strong linear increase is present in all altitude/latitude bins and varies considerably.

Figure 18 summarizes the derived decadal trends for all latitude/altitude bins. The trend is positive for all latitude and altitude bins and highly significant (significance of $5 \sigma$ or more). In the lower stratosphere below $20 \mathrm{~km}$ the trend is between 40 and $50 \%$ per decade, while it varies between $30 \%$ per decade in the northern middle and high latitudes between 20 and $30 \mathrm{~km}, 50$ to $60 \%$ per decade in the southern midlatitudes from 20 to $50 \mathrm{~km}$, and 70 to more than $100 \%$ per decade in the northern upper stratosphere at middle and high latitudes. The maximum of absolute trends is in the northern subtropics around $15 \mathrm{~km}$, supporting our hypothesis that this region is fed by the strongly increasing East Asian emissions. In contrast, the relative trends are not highest in this region, due to the strongly enhanced volume mixing ratios measured there.

Recent HCFC-22 trends derived by earlier works for various time periods, altitudes and latitudes are summarised in Table 3 and compared to MIPAS-derived trends. It has to be noted that the relative trends are based on varying reference vmrs, most of which are not even reported. For this reason we discuss here the comparison of absolute trends only. In general, the trends derived from MIPAS observations are higher than the trends already published. The only exception are the trends at high southern latitudes by Moore and Remedios (2008) that were derived from a combination of ATMOS and MIPAS observations, and that are somewhat higher than our MIPAS-derived trends. The higher trends found by MIPAS reflect the increase of the surface growth rates during the MIPAS mission period.

\subsection{Comparisons between upper tropospheric and surface growth rates}

Two networks perform regular, long-term and highly precise near-surface measurements of various tracers, among them HCFC-22: these are the NOAA Earth System Research Laboratory (ESRL) Global Monitoring Division (GMD) and the Advanced Global Atmospheric Gases Experiment (AGAGE). Although not directly comparable, because MIPAS observations do not reach the ground, we compare here mean tropospheric values (below $10 \mathrm{~km}$ altitude) re-
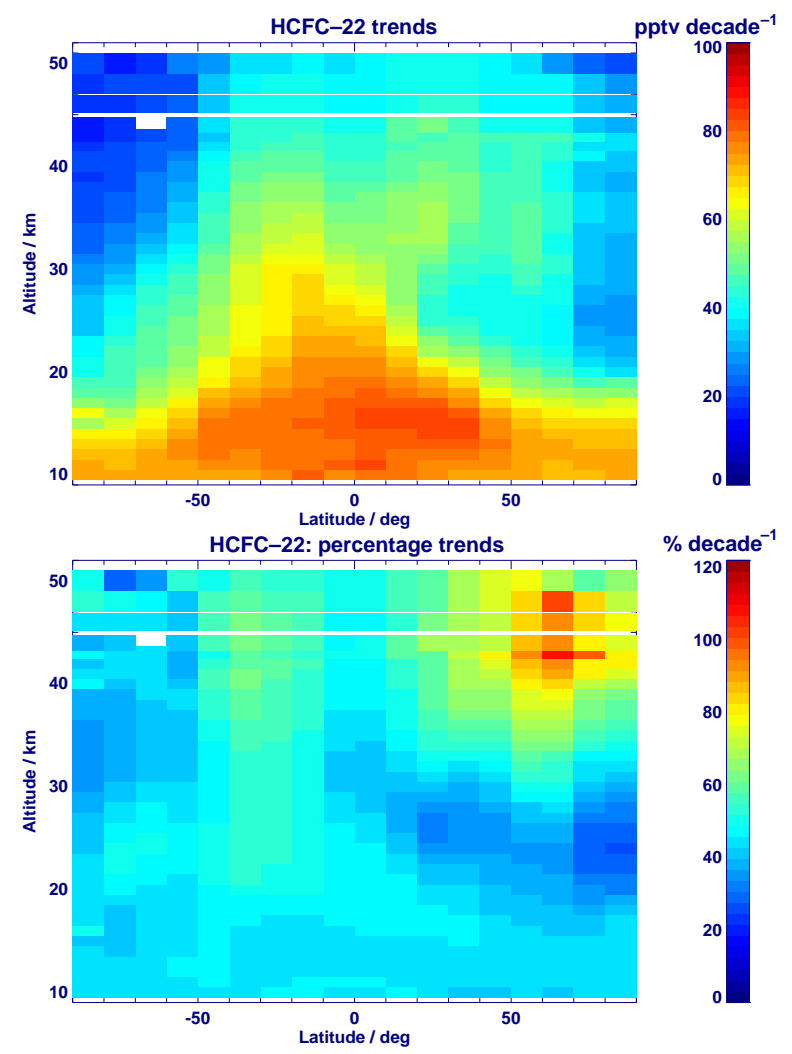

Figure 18. Trend of HCFC-22 volume mixing ratio for all latitude/altitude bins, in absolute units (pptvdecade ${ }^{-1}$ ) (top) and as percentage per decade relative to the background distribution in 2005 (i.e. the constant term of the regression analysis, see Eq. 1) (bottom). For the percentage trends, autocorrelations have been considered in the fit.

trieved from MIPAS data with the surface observations from NOAA/GMD and AGAGE, making use of the fact that within each hemisphere the free troposphere can be considered well-mixed in the altitude domain. This assumption is confirmed by aircraft measurements (Xiang et al., 2014). In this context it should be noted that surface measurements are reported as dry air mole fraction, while MIPAS measurements are reported in mixing ratios where the air with its actual water vapour content is the reference. Since the stratosphere is very dry where MIPAS measures, this makes no discernable difference. Near surface, however, this difference has to be taken into account but since air dries during uplift, the surface dry air mole fraction is exactly the quantity which is comparable to the MIPAS results.

\subsubsection{The surface networks}

\section{NOAA/GMD}

NOAA/GMD runs flask measurements at remote site since 1992 (Montzka et al., 2009, 2015). These data are reported on the NOAA-2006 scale. These data are available 
Table 3. Recent trends of HCFC-22 depending on time period, altitude and latitude from earlier publications, compared to MIPAS-derived trends at the respective altitude/latitude.

\begin{tabular}{|c|c|c|c|c|c|c|}
\hline Source & Time interval & Latitude/altitude & $\begin{array}{l}\text { Absolute trend } \\
{\left[\text { pptv year }^{-1}\right]}\end{array}$ & $\begin{array}{r}\text { Relative trend } \\
{\left[\% \text { year }^{-1}\right]^{\mathrm{a}}}\end{array}$ & $\begin{array}{r}\text { MIPAS-derived } \\
\text { trend [pptv year }{ }^{-1} \text { ] }\end{array}$ & $\begin{array}{l}\text { MIPAS-derived } \\
\text { trend }\left[\% \text { year }^{-1}\right]\end{array}$ \\
\hline \multirow[t]{2}{*}{ Moore and Remedios (2008) } & 1994-2004 & $20-50^{\circ} \mathrm{N} / 20 \mathrm{~km}$ & $5.4 \pm 0.7$ & $3.5 \pm 0.4^{\mathrm{b}}$ & $6.15 \pm 0.24$ & $4.24 \pm 0.16$ \\
\hline & 1994-2003 & $60-80^{\circ} \mathrm{S} / 20 \mathrm{~km}$ & $6.0 \pm 0.7$ & $4.3 \pm 0.5^{\mathrm{b}}$ & $4.88 \pm 0.10$ & $4.55 \pm 0.09$ \\
\hline \multirow[t]{3}{*}{ Rinsland et al. (2005a) } & 1985 & $30^{\circ} \mathrm{N} /$ lower strat. & & $14.57 \pm 4.1^{\mathrm{c}}$ & & \\
\hline & 1994 & $30^{\circ} \mathrm{N} /$ lower strat. & & $6.35 \pm 2.24^{\mathrm{c}}$ & & \\
\hline & 2004 & $30^{\circ} \mathrm{N} /$ lower strat. & & $3.92 \pm 2.08^{\mathrm{c}}$ & $7.60 \pm 0.21$ & $4.56 \pm 0.12$ \\
\hline Rinsland et al. (2005b) & $1987-2002$ & $30^{\circ} \mathrm{N} / 2-10 \mathrm{~km}$ & $5.66 \pm 0.15$ & $6.47 \pm 0.17^{\mathrm{b}}$ & $7.54 \pm 1.53$ & $4.32 \pm 0.87$ \\
\hline Brown et al. (2011) & 2004-2010 & $30^{\circ} \mathrm{S}-30^{\circ} \mathrm{N} / 8-17 \mathrm{~km}$ & $6.56 \pm 0.20$ & $3.7 \pm 0.1^{\mathrm{b}}$ & $8.02 \pm 0.03$ & $4.48 \pm 0.02$ \\
\hline
\end{tabular}

a The reference vmrs on which the relative trends are based vary from data set to data set. ${ }^{\mathrm{b}}$ Linear fit. ${ }^{\mathrm{c}}$ Derivatives at the respective years from a parabolic fit.

via ftp://ftp.cmdl.noaa.gov/hats/hcfcs/hcfc22/flasks/. Global HCFC-22 data from NOAA/GMD in units of mole fraction in dry air at ground level are shown in Fig. 19 for various measurement sites from the South Pole to the high Arctic. The data are not filtered for any pollution events, resulting in some enhanced values for the stations Trinidad Head and Mace Head that are occasionally influenced by nearby emissions. The MIPAS HCFC-22 monthly mean values for latitude bands selected to match the latitudes of the stations, averaged over all altitudes below $10 \mathrm{~km}$, are shown for comparison with the same colour code as the respective station data.

\section{AGAGE}

AGAGE provides in situ measurements of a wide range of ozone depleting compounds and greenhouse gases, including HCFC-22, from several ground stations (Prinn et al., 2000, 2013; O'Doherty et al., 2004). AGAGE measurements used here are obtained using in situ gas chromatography with mass spectrometry (GC-MS) detection technique and are reported on the SIO-2005 calibration scale. NOAA flask results and AGAGE in situ data are compared every 6 months at common sites. Comparison of HCFC-22 from NOAA flasks, using the NOAA-2006 calibration scale to AGAGE in situ measurements based on the SIO-2005 calibration scale at Cape Grim, Samoa, Trinidad Head and Mace Head reveal the following differences: the average difference (NOAA minus AGAGE) across the four sites is $-0.7 \pm 0.5 \mathrm{ppt}$ or $0.35 \pm 0.25 \%$. The differences are also relatively constant with time. These results are also very consistent to those found in the International Halocarbons in Air Comparison Experiment (Hall et al., 2014).

\subsubsection{Comparison with MIPAS}

Despite quite different approaches to making the measurements (in situ high frequency at relatively few sites compared to low frequency flask measurements at more sites), the independent surface data provided by NOAA/GMD and AGAGE show the same broad features, distributions, seasonality and trends. Thus, both data sets are discussed relative to MIPAS results together.

Overall, surface data from both networks and MIPAS upper tropospheric mixing ratios of HCFC-22 show a good agreement regarding the trends (Fig. 19). The interhemispheric differences of the surface data are clearly visible, with mole fractions in the Northern Hemisphere being larger by about $10 \%$ than $\mathrm{SH}$ values, due to the main sources of HCFC-22 being located in the NH. MIPAS upper tropospheric mixing ratios agree best with tropical $\mathrm{NH}$ surface measurements, reflecting the fact that uplift of surface air is dominated by tropical processes.

In the $\mathrm{SH}$, tropospheric MIPAS values are mostly significantly higher than the surface values at the same latitudes and reach these, if any, only during their seasonal minima (Fig. 19, top and middle panel). The reason is roughly this. MIPAS, whose measurements refer to the upper troposphere and above, sees more advected air from the tropical outflow, and the signal is modulated by a pronounced seasonal cycle, while the related ground-based measurements are clean-air measurements. This hypothesis is in tendency confirmed by SH aircraft measurements (e.g. Xiang et al., 2014), where an indication of higher mixing ratios at higher altitudes is found, which is attributed to transport of $\mathrm{NH}$ air into the $\mathrm{SH}$ at higher altitudes in lower latitudes. It is interesting to see that among the SH time series, the seasonal cycle in MIPAS data is strongest for the southern polar latitude band (see Fig. 19, top panel), while the minima reached within this time series at the end of Antarctic summer are the lowest among all latitude bands. This strong seasonal cycle is also visible in Fig. 16 with maxima in polar southern spring and indicates flooding of the southern polar UTLS (Upper Troposphere / Lower Stratosphere) region with low-latitude air around the time of the polar vortex breakdown.

The MIPAS measurements show smaller differences between the hemispheres than the surface measurements. This is explained by the fact that MIPAS observes air at altitudes where the outflow of the tropical convergence contributes to the composition of air. The assumption that air uplifted within the tropical convergence region is mixed be- 

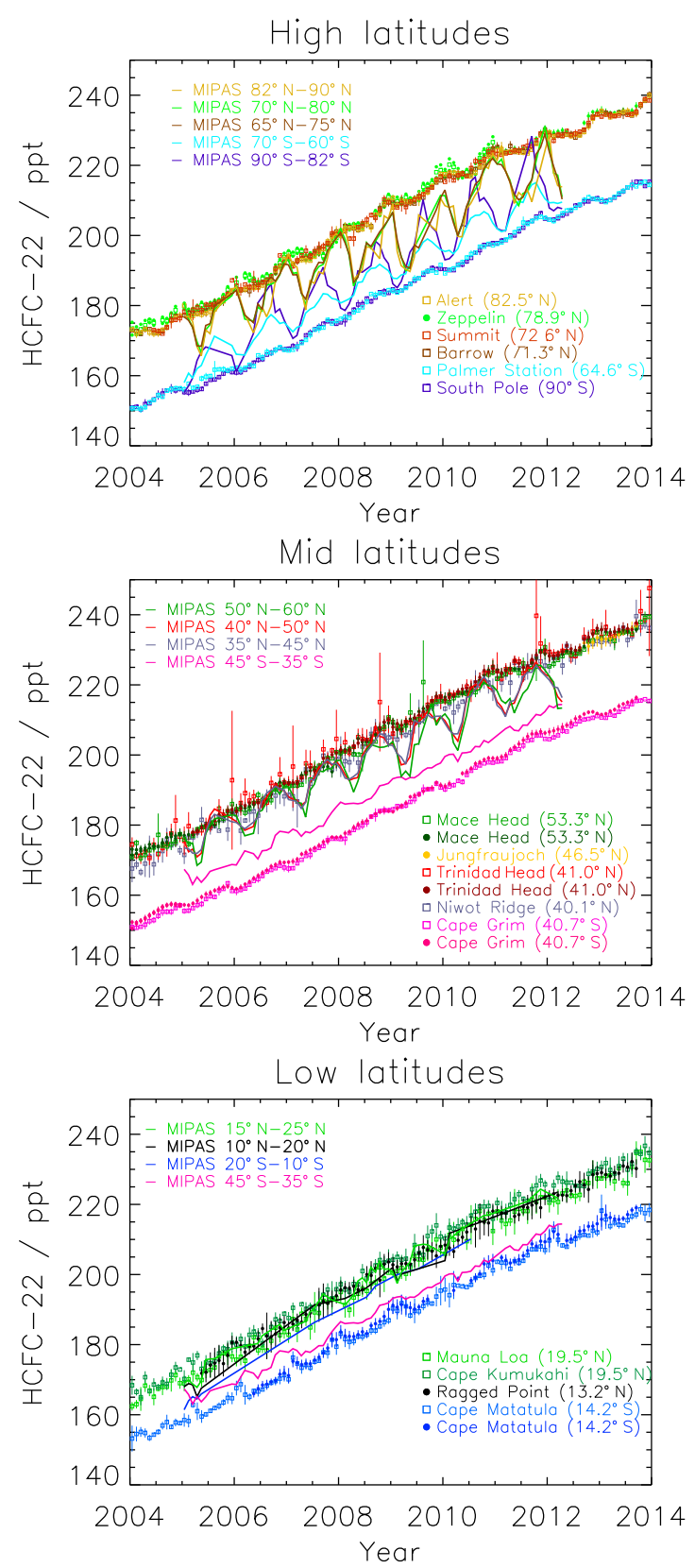

Figure 19. Comparison of HCFC-22 time series from several stations of the NOAA/GMD program and the AGAGE program with corresponding MIPAS values. Open squares: NOAA/GMD monthly mean data from several stations (see legend for colour code); filled circles: AGAGE monthly mean data from several stations (see legend for colour code); solid lines without symbols: MIPAS monthly mean data for $10^{\circ}$ latitude bands around the respective station latitude and averaged over data points for altitudes below $10 \mathrm{~km}$; the colour coding matches that of the stations. The tick marks at the horizontal axis mark the beginning of the years. (top) High latitudes (between 60 and $90^{\circ} \mathrm{N}-\mathrm{S}$ ); (middle) mid-latitudes (between $30^{\circ}$ and $60^{\circ} \mathrm{N}-\mathrm{S}$ ); (bottom) low latitudes (between $30^{\circ} \mathrm{S}$ and $30^{\circ} \mathrm{N}$.) tween both hemispheres offers an explanation for the reduced hemispheric contrast in the MIPAS data. Also these findings are in agreement with those of (Xiang et al., 2014).

Finally, the most obvious difference between surface time series and zonally averaged MIPAS upper troposphere time series is the pronounced seasonal cycle in the latter, with minimum values during $\mathrm{NH}$ spring and $\mathrm{SH}$ summer (see Fig. 19, top and middle panel). It is far more pronounced in the $\mathrm{NH}$ mid-latitudes than in the $\mathrm{SH}$ mid-latitudes and its amplitude increases towards high latitudes.

Xiang et al. (2014) observed a seasonality in the surface measurements of HCFC-22 with minima in northern summer and attributed this to increased scavenging through the $\mathrm{OH}$ radical reaction and seasonality in the transport. The best explanation of the observed seasonality, however, relies on an additional seasonality of the emissions of refrigerants with maxima in summer. This seasonality of emissions was derived by inverse modelling of Xiang et al. (2014) aircraft measurements.

MIPAS maximum values are in excellent agreement with those of surface stations in the $\mathrm{NH}$. While $\mathrm{OH}$ scavenging and seasonal variations in transport could possibly explain the summer minimum observed by MIPAS in the $\mathrm{SH}$, the springtime minimum in the $\mathrm{NH}$ is more probably related to intrusion of HCFC-22-poor stratospheric air at the end of the polar winter and during polar vortex breakdown. Similar springtime minima were also observed for other tropospheric source gases and have been attributed to stratospheric air intrusions (Nevison et al., 2004, 2011). The fact that the minima in the UTLS in the MIPAS time series at higher altitudes precede those at lower altitudes (cf. Fig 15 bottom right panel and Fig. 16, top two panels) supports this. The amplitude of the seasonal cycle seems to be even more enhanced due to transport of high HCFC-22 abundances uplifted within the Asian monsoon anticyclone to higher latitudes during fall (compare Fig. 16, top left panel).

The SH upper tropospheric annual cycle is not exactly in the opposite phase of its northern counterpart. The latitudes of the SH stations shown in this comparison are, however, generally lower than the latitudes of the $\mathrm{NH}$ stations. At these low latitudes the upper tropospheric air is not modulated that much by polar processes but by the change of the position of the intertropical convergence zone and the Hadley cell. In local summer these latitudes are affected by the outflow of the tropical convergence, providing young HCFC-22-rich air. This hypothesis is corroborated by the fact that the MIPAS time series at $45-35^{\circ} \mathrm{S}$ (pink solid line in Fig. 19) follows the Cape Matatula (Samoa) (14.2 $2^{\circ} \mathrm{S}$, blue open squares and filled circles) time series remarkably well, while it appears to be uncorrelated with the Cape Grim (Tasmania) $\left(40.7^{\circ} \mathrm{S}\right)$ data (pink open squares and filled circles).

While according to MIPAS the HCFC-22 global mean below $10 \mathrm{~km}$ altitude (not shown) increased from 161 to 211 pptv between January 2005 and April 2012, the NOAA ground-based global mean for these months were 164 and 
$216 \mathrm{pptv}$, providing an increase of 50 (MIPAS) and 52 (NOAA) ppt, respectively. The growth rate derived from NOAA/GMD data for the NH is only slightly higher than that inferred from MIPAS measurements below $10 \mathrm{~km}(52$ vs. 49 ppt over 7 years).

\subsection{Unexplained stratospheric trends}

According to Kellmann et al. (2012) we call differences between the MIPAS stratospheric percentage trends (relative to the HCFC-22 vmr in 2005) and the tropospheric percentage trends corrected for age of stratospheric air "unexplained trends"; these are shown in Fig. 20, bottom panel. The top panel gives a schematic sketch of the method to derive the unexplained trends: we calculated surface trends for periods of similar length as the MIPAS observation period from NOAA/GMD global mean data (black bullets in the top panel of Fig. 20; black horizontal bars indicate the periods for which these trends have been derived; the vertical bars are the uncertainties of the surface trends). The MIPAS stratospheric trends (coloured bullets for three selected latitude/altitude bins) are compared to the surface trends. However, the time assignment of the MIPAS trends was corrected for the age of stratospheric air. The coloured horizontal arrows in the figure represent the corrective shift in time. This is, we compare to the surface trends at the time the respective air parcel left the troposphere. Age of air information is taken from Stiller et al. (2012). The difference between the trend in the stratosphere observed by MIPAS and the trend at the surface at the time when the air parcel started its travel into the stratosphere is understood as unexplained trend. We analyse relative (percentage) trends instead of absolute trends because any losses in the stratosphere are proportional to the abundances, thus they would change the trends in absolute units, but leave relative trends unaffected. Any difference between the AoAcorrected relative surface trends and the relative trends seen by MIPAS cannot come from chemical reactions, but must be due to changes of the transport patterns. For a stationary residual circulation, the surface trends should be mapped to the stratosphere according to the stratospheric AoA distribution, resulting in zero unexplained trends all over the stratosphere. However, the pattern of unexplained trends as shown in Fig. 20, bottom panel indicate that a stationary residual circulation was not the case over the observation period of MIPAS. The unexplained trends are considered significant if they are larger than their $2 \sigma$ uncertainties, which is the case for the pronounced deviations from zero. Uncertainties were assessed via Gaussian error propagation of the uncertainties of the measurement data (for NOAA/GMD data: uncertainties of the monthly global mean data as provided; for MIPAS HCFC-22 vmrs: standard error of the monthly zonal mean data; for age of air: an overall uncertainty of 0.5 years was assumed).

We find positive and negative trend differences of up to 40 percent. The HCFC-22 trend in the northern mid-
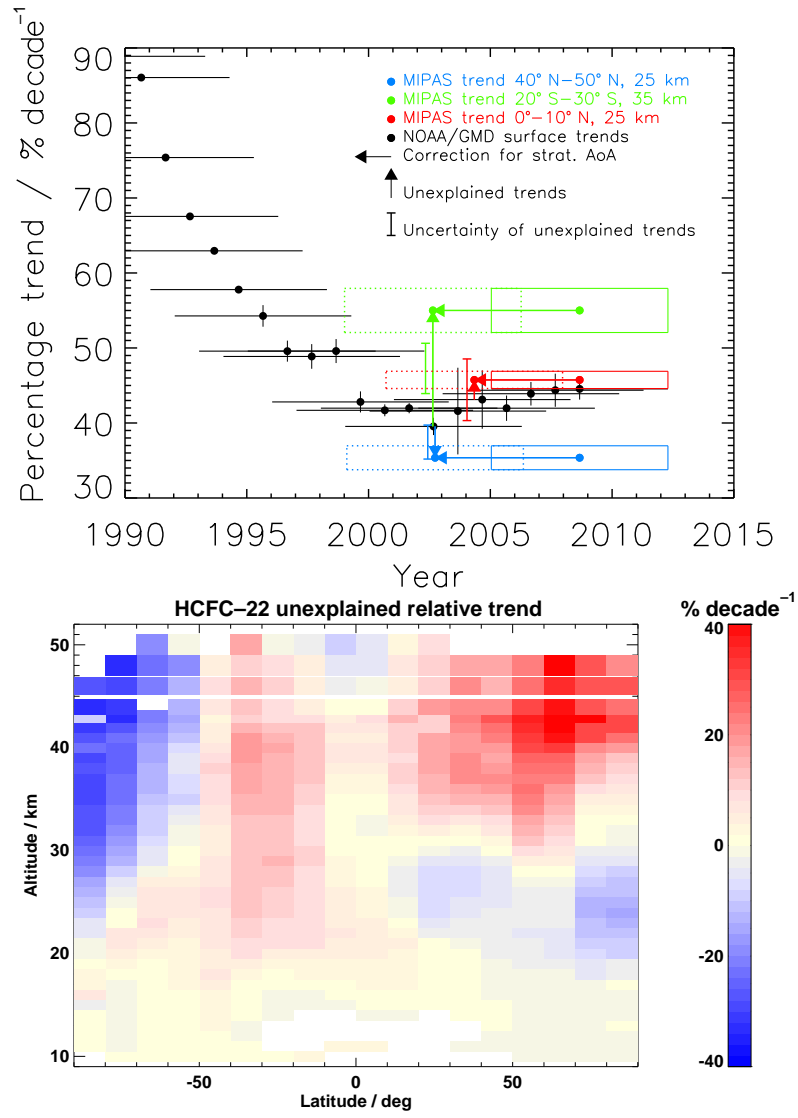

Figure 20. (top) Schematic sketch of the procedure to derive unexplained trends. The black bullets with the vertical error bars are the relative trends derived from NOAA/GMD global mean surface data for the periods indicated by the horizontal bars. The relative trends are given as percentage change relative to the HCFC-22 volume mixing ratios at the beginning of the respective period, similar to the MIPAS relative trends. For the MIPAS relative trends (referenced to volume mixing ratios determined for January 2005) three examples for three different latitude/altitude bins are given (coloured bullets). The boxes around the bullets give the uncertainties of the trends (vertical direction) and the period these trends are valid for (horizontal direction, MIPAS observation period). The horizontal arrows indicate the stratospheric age of air for the respective latitude/altitude bin used within the comparison to surface trends. The AoA information is from Stiller et al. (2012). The vertical arrows indicate the unexplained trends, i.e. the differences between the MIPAS trends and the surface trends at the time the stratospheric air parcels left the troposphere. The error bars plotted along with the unexplained trends provide their $2 \sigma$ uncertainty; the unexplained trends are considered significant if they are larger than their error bars. (bottom) Unexplained stratospheric HCFC-22 trends, i.e. differences between MIPAS stratospheric relative trends (referenced to the HCFC-22 distribution in January 2005, i.e. the constant term of the regression analysis (see Eq. 1) and relative trends at the surface measured by the NOAA/GMD program (global means), the latter corrected for the time lag between the air parcels' start in the troposphere and its arrival in the stratosphere. 
latitude lower stratosphere $(15-30 \mathrm{~km}$ ) is slightly smaller (up to $-10 \%$ ) than the trend at the ground at the time the air started its travel into the stratosphere, while in the southern mid-latitudes, the HCFC-22 trend is larger than the trend observed at surface level over all the stratosphere. In the polar regions, the trend is up to $40 \%$ lower at the South Pole, and up to $40 \%$ higher at the North Pole and the northern midlatitudes. This pattern of trend differences is in agreement with the patterns found for CFC-11 and CFC-12 (Kellmann et al., 2012), and is in accordance with the AoA trends found by Stiller et al. (2012).

Positive AoA trends in the northern mid-latitudes below $30 \mathrm{~km}$ and the southern polar region lead to increasingly longer exposure of HCFC-22 to loss processes in the stratosphere, and thus to a reduction of the positive HCFC-22 trend, while the general positive HCFC-22 trend is further increased in regions where the AoA becomes younger, leaving less time for stratospheric HCFC-22 loss processes. It should be noted that any incorrect assumption on AoA as such, used within the correction of surface trends, cannot explain both the positive and negative signs in the trend differences. Any incorrect assumption on the AoA would lead to incorrect differences, which, however, had always the same positive or negative sign. Thus, the occurrence of positive and negative trend differences is an unambiguous sign that the circulation must have changed over the MIPAS observation period. Our results further show that these circulation changes were different in the northern and southern mid-latitudes, as was concluded from the AoA data inferred from the MIPAS $\mathrm{SF}_{6}$ data record (Stiller et al., 2012). These results confirm the hypothesis of Stiller et al. (2012) of interhemispheric asymmetries in circulation changes, for which further evidence has been found by Eckert et al. (2014) (interhemispheric asymmetries in decadal MIPAS ozone trends), Nedoluha et al. (2015) (interhemispheric asymmetries in decadal trends of $\mathrm{N}_{2} \mathrm{O}$ from the Microwave Limb Sounder (MLS)), and Mahieu et al. (2014) ( $\mathrm{HCl}$ trends and modelled age-of-air trends).

\section{Summary and conclusions}

HCFC-22 data from MIPAS for the period 2005 to 2012 were produced and analysed. Version 5 level- $1 \mathrm{~b}$ reduced resolution MIPAS measurements (nominal mode) of the period from 27 January 2005 to 8 April 2012 were inverted using the MIPAS IMK/IAA scientific data processor. The profile retrieval was performed by Tikhonov-constrained non-linear least squares fitting of measured limb spectral radiances. The total error of the retrieval is $7 \%$ on $20 \mathrm{~km}$ height and $9 \%$ on $30 \mathrm{~km}$ height, and the error budget is dominated by noise. A typical retrieved profile represents approximately $5^{\circ}$ of freedom, corresponding to an altitude resolution of typically between $3 \mathrm{~km}$ at $10 \mathrm{~km}$ height and $7 \mathrm{~km}$ at $30 \mathrm{~km}$ height, further increasing with height. The percentage of non-converged profiles is about 0.01 to $0.02 \%$.
The profiles obtained were compared with ACE-FTS satellite data (v3.5), as well as with MkIV balloon profiles and in situ measurements performed by the University of Frankfurt. The comparisons are ambiguous with respect to a bias of MIPAS measurements; in general we can state that MIPAS agrees within \pm 15 pptv $(1 \sigma)$ with ACE-FTS and MkIV reference measurements. Between 13 and $22 \mathrm{~km}$, good agreement with MkIV and ACE-FTS profiles is demonstrated. A high bias of 30-50 pptv relative to cryosampler measurements was found below $24 \mathrm{~km}$ but no bias was found for higher altitudes.

The global distribution of HCFC-22 vmr reflects the mean circulation in the stratosphere and reveals also seasonal oscillations. The HCFC-22 annual cycle in the $\mathrm{SH}$ is more pronounced than in the NH. The HCFC-22 volume mixing ratio in the $\mathrm{NH}$ troposphere is generally higher than in the $\mathrm{SH}$, due to main emission sources residing there. A volume mixing ratio maximum is situated at about $16 \mathrm{~km}$ height at low latitudes, which exceeds mixing ratios measured at all remote surface sites in the $\mathrm{NH}$ or $\mathrm{SH}$. We attribute this to advection to low latitudes of HCFC-22-rich air uplifted in the Asian monsoon area. The source of this HCFC-22-rich air could be South-East Asia. United Nations Environment Programme (2012) reports that China has been the largest global producer and consumer of HCFCs for a number of years. In the upper troposphere, monsoon air rich in HCFC-22 is instantaneously mixed into the tropics.

A multi-variate regression analysis was performed for $10^{\circ}$ latitude $/ 1-2 \mathrm{~km}$ altitude bins, with terms for linear variations, sinusoidal variations with various periods, and a proxy for the QBO variation. We find positive linear trends for all latitude/altitude bins, ranging from $83 \mathrm{pptv} \mathrm{decade}^{-1}$ in the northern subtropical lower stratosphere to $18 \mathrm{pptv}$ decade $^{-1}$ in the southern polar upper stratosphere. The absolute trends are in the range of previous analyses. Percentage trends are highest in the southern mid-latitudinal stratosphere (50$70 \%$ decade $\left.^{-1}\right)$ and in the northern polar upper stratosphere (70 to $120 \%$ decade $^{-1}$ ) and lowest in the northern lower stratosphere (around $30 \% \mathrm{decade}^{-1}$ ). The highest seasonal amplitudes are observed in the $60^{\circ}$ regions; in the tropical lower stratosphere the amplitudes are low.

Global NOAA/GMD, AGAGE and MIPAS tropospheric values show good absolute agreement and similar trends. Based on the absolute values of HCFC-22 from MIPAS and on the MIPAS derived HCFC-22 growth rate, one can conclude that the HCFC-22 global volume mixing ratio in the lower stratosphere has risen by 49 pptv in 7 years. A pronounced seasonality has been detected in the upper troposphere with minima in spring in the NH and in local summer in the SH. The latter is attributed to the seasonality of the main tropical uplift and outflow regions. The seasonality in the NH is attributed to the intrusion of HCFC-22-poor stratospheric air at the end of the Arctic winter. Inconsistencies in percentage trends between ground-based and agecorrected MIPAS stratospheric data hint at recent changes in 
stratospheric circulation. Similar indication has been found by analysis of trends of the mean AoA (Stiller et al., 2012), ozone (Eckert et al., 2014) and CFC-11 as well as CFC-12 (Kellmann et al., 2012). A more detailed analysis of these circulation changes is currently under investigation.

MIPAS HCFC-22 data presented here can be downloaded after registration from http://www.imk-asf.kit.edu/english/ 308.php. The HCFC-22 trends shown in Figs. 18 and 20 are provided as numerical values in the Supplement.

\section{The Supplement related to this article is available online at doi:10.5194/acp-16-3345-2016-supplement.}

Acknowledgements. We acknowledge provision of MIPAS level-1b data by ESA. NOAA measurements of HCFC-22 are made possible in part by funding from the NOAA Climate Program Office's AC4 program. Standards, flask handling and flask analysis at NOAA are provided with assistance from B. Hall, C. Siso and D. Mondeel. AGAGE is supported principally by NASA (USA) grants to MIT and SIO and also by the following: DECC (UK) and NOAA (USA) grants to Bristol University; CSIRO and the Bureau of Meteorology (Australia); FOEN grants to Empa (Switzerland); NILU (Norway); SNU (Korea); CMA (China); NIES (Japan); and Urbino University (Italy). Part of this research was performed at the Jet Propulsion Laboratory, California Institute of Technology, under contract with NASA. We thank the Columbia Scientific Balloon Facility (CSBF) for performing the launches of the JPL MkIV instrument. The Atmospheric Chemistry Experiment (ACE), also known as SCISAT, is a Canadian-led mission mainly supported by the Canadian Space Agency and the Natural Sciences and Engineering Research Council of Canada. Data analysis at IMK has been supported by BMBF under contract number 50EE0901. The authors thank three reviewers and the editor for their constructive as well as critical comments that helped to improve the paper. We acknowledge support by Deutsche Forschungsgemeinschaft and Open Access Publishing Fund of Karlsruhe Institute of Technology.

The article processing charges for this open-access

publication were covered by a Research

Centre of the Helmholtz Association.

Edited by: P. Liebing

\section{References}

Bernath, P. F., McElroy, C. T., Abrams, M. C., Boone, C. D., Butler, M., Camy-Peyret, C., Carleer, M., Clerbaux, C., Coheur, P.F., Colin, R., DeCola, P., De Mazière, M., Drummond, J. R., Dufour, D., Evans, W. F. J., Fast, H., Fussen, D., Gilbert, K., Jennings, D. E., Llewellyn, E. J., Lowe, R. P., Mahieu, E., McConnell, J. C., McHugh, M., McLeod, S. D., Michaud, R., Midwinter, C., Nassar, R., Nichitiu, F., Nowlan, C., Rinsland, C. P., Rochon, Y. J., Rowlands, N., Semeniuk, K., Simon, P., Skelton, R., Sloan, J. J., Soucy, M.-A., Strong, K., Tremblay, P.,
Turnbull, D., Walker, K. A., Walkty, I., Wardle, D. A., Wehrle, V., Zander, R., and Zou, J.: Atmospheric Chemistry Experiment (ACE): Mission overview, Geophys. Res. Lett., 32, L15S01, doi:10.1029/2005GL022386, 2005.

Birner, T. and Bönisch, H.: Residual circulation trajectories and transit times into the extratropical lowermost stratosphere, Atmos. Chem. Phys., 11, 817-827, doi:10.5194/acp-11-817-2011, 2011.

Bönisch, H., Engel, A., Birner, Th., Hoor, P., Tarasick, D. W., and Ray, E. A.: On the structural changes in the Brewer-Dobson circulation after 2000, Atmos. Chem. Phys., 11, 3937-3948, doi:10.5194/acp-11-3937-2011, 2011.

Boone, C. D., Nassar, R., Walker, K. A., Rochon, Y., McLeod, S. D., Rinsland, C. P., and Bernath, P. F.: Retrievals for the atmospheric chemistry experiment Fourier-transform spectrometer, Appl. Optics, 44, 7218-7231, 2005.

Boone, C. D., Walker, K. A., and Bernath, P. F.: Version 3 Retrievals for the Atmospheric Chemistry Experiment Fourier Transform Spectrometer (ACE-FTS), in: The Atmospheric Chemistry Experiment ACE at 10: A Solar Occultation Anthology, edited by: Bernath, P. F., 103-127, A. Deepak Publishing, Hampton, Virginia, USA, 2013.

Brown, A. T., Chipperfield, M. P., Boone, C., Wilson, C., Walker, K. A., and Bernath, P. F.: Trends in atmospheric halogen containing gases since 2004, J. Quant. Spectrosc. Ra., 112, 2552-2566, doi:10.1016/j.jqsrt.2011.07.005, 2011.

Carlotti, M.: Global-fit approach to the analysis of limb-scanning atmospheric measurements, Appl. Optics, 27, 3250-3254, 1988.

Connor, B. J., Siskind, D. E., Tsou, J. J., Parrish, A., and Remsberg, E. E.: Ground-based microwave observations of ozone in the upper stratosphere and mesosphere, J. Geophys. Res., 99, 1675716770, doi:10.1029/94JD01153, 1994.

Eckert, E., von Clarmann, T., Kiefer, M., Stiller, G. P., Lossow, S., Glatthor, N., Degenstein, D. A., Froidevaux, L., GodinBeekmann, S., Leblanc, T., McDermid, S., Pastel, M., Steinbrecht, W., Swart, D. P. J., Walker, K. A., and Bernath, P. F.: Drift-corrected trends and periodic variations in MIPAS IMK/IAA ozone measurements, Atmos. Chem. Phys., 14, 25712589, doi:10.5194/acp-14-2571-2014, 2014.

Eckert, E., Laeng, A., Lossow, S., Kellmann, S., Stiller, G., von Clarmann, T., Glatthor, N., Höpfner, M., Kiefer, M., Oelhaf, H., Orphal, J., Funke, B., Grabowski, U., Haenel, F., Linden, A., Wetzel, G., Woiwode, W., Bernath, P. F., Boone, C., Dutton, G. S., Elkins, J. W., Engel, A., Gille, J. C., Kolonjari, F., Sugita, T., Toon, G. C., and Walker, K. A.: MIPAS IMK/IAA CFC-11 $\left(\mathrm{CCl}_{3} \mathrm{~F}\right)$ and $\mathrm{CFC}-12\left(\mathrm{CCl}_{2} \mathrm{~F}_{2}\right)$ measurements: accuracy, precision and long-term stability, Atmos. Meas. Tech. Discuss., 8 , 7573-7662, doi:10.5194/amtd-8-7573-2015, 2015.

Endemann, M. and Fischer, H.: Envisat's High-Resolution Limb Sounder: MIPAS, ESA bulletin, 76, 47-52, 1993.

Endemann, M., Gare, P., Smith, D., Hoerning, K., Fladt, B., and Gessner, R.: MIPAS Design Overview and Current Development Status, in: Proceedings EUROPTO series, Optics in Atmospheric Propagation, adaptive systems, and Lidar techniques for Remote Sensing, Taormina, Italy, 24-26 September, 1996, Vol. 2956, 124-135, 1996.

Engel, A., Schmidt, U., and Stachnik, R. A.: Partitioning between Chlorine Reservoir Species deduced from Observations in the Arctic Winter stratosphere, J. Atmos. Chem., 27, 107-126, 1997. 
European Space Agency: Envisat, MIPAS An instrument for atmospheric chemistry and climate research, ESA Publications Division, ESTEC, P.O. Box 299, 2200 AG Noordwijk, the Netherlands, SP-1229, 2000.

Fischer, H. and Oelhaf, H.: Remote sensing of vertical profiles of atmospheric trace constituents with MIPAS limb-emission spectrometers, Appl. Optics, 35, 2787-2796, 1996.

Fischer, H., Birk, M., Blom, C., Carli, B., Carlotti, M., von Clarmann, T., Delbouille, L., Dudhia, A., Ehhalt, D., Endemann, M., Flaud, J. M., Gessner, R., Kleinert, A., Koopman, R., Langen, J., López-Puertas, M., Mosner, P., Nett, H., Oelhaf, H., Perron, G., Remedios, J., Ridolfi, M., Stiller, G., and Zander, R.: MIPAS: an instrument for atmospheric and climate research, Atmos. Chem. Phys., 8, 2151-2188, doi:10.5194/acp-8-2151-2008, 2008.

Gardiner, T., Forbes, A., de Mazière, M., Vigouroux, C., Mahieu, E., Demoulin, P., Velazco, V., Notholt, J., Blumenstock, T., Hase, F., Kramer, I., Sussmann, R., Stremme, W., Mellqvist, J., Strandberg, A., Ellingsen, K., and Gauss, M.: Trend analysis of greenhouse gases over Europe measured by a network of ground-based remote FTIR instruments, Atmos. Chem. Phys., 8, 6719-6727, doi:10.5194/acp-8-6719-2008, 2008.

Goldman, A., Murcray, F. J., Blatherwick, R. D., Bonomo, F. S., Murcray, F. H., and Murcray, D. G.: Spectroscopic identification of $\mathrm{CHClF}_{2}(\mathrm{~F}-22)$ in the lower stratosphere, Geophys. Res. Lett., 8, 1012-1014, doi:10.1029/GL008i009p01012, 1981.

Hall, B. D., Engel, A., Mühle, J., Elkins, J. W., Artuso, F., Atlas, E., Aydin, M., Blake, D., Brunke, E.-G., Chiavarini, S., Fraser, P. J., Happell, J., Krummel, P. B., Levin, I., Loewenstein, M., Maione, M., Montzka, S. A., O’Doherty, S., Reimann, S., Rhoderick, G., Saltzman, E. S., Scheel, H. E., Steele, L. P., Vollmer, M. K., Weiss, R. F., Worthy, D., and Yokouchi, Y.: Results from the International Halocarbons in Air Comparison Experiment (IHALACE), Atmos. Meas. Tech., 7, 469-490, doi:10.5194/amt-7469-2014, 2014.

IPCC: Climate Change 2014: Mitigation of Climate Change. Contribution of Working Group III to the Fifth Assessment Report of the Intergovernmental Panel on Climate Change, edited by: Edenhofer, O., Pichs-Madruga, R., Sokona, Y., Farahani, E., Kadner, S., Seyboth, K., Adler, A., Baum, I., Brunner, S., Eickemeier, P., Kriemann, B., Savolainen, J., Schlömer, S., von Stechow, C., Zwickel, T., and Minx, J. C., Cambridge University Press, Cambridge, United Kingdom and New York, NY, USA, 2014.

Kellmann, S., von Clarmann, T., Stiller, G. P., Eckert, E., Glatthor, N., Höpfner, M., Kiefer, M., Orphal, J., Funke, B., Grabowski, U., Linden, A., Dutton, G. S., and Elkins, J. W.: Global CFC-11 $\left(\mathrm{CCl}_{3} \mathrm{~F}\right)$ and CFC-12 $\left(\mathrm{CCl}_{2} \mathrm{~F}_{2}\right)$ measurements with the Michelson Interferometer for Passive Atmospheric Sounding (MIPAS): retrieval, climatologies and trends, Atmos. Chem. Phys., 12, 11857-11875, doi:10.5194/acp-12-11857-2012, 2012.

Kiefer, M., von Clarmann, T., and Grabowski, U.: State parameter Data Base for MIPAS Data Analysis, Adv. Space Res., 30, $2387-$ 2392, 2002.

Kiefer, M., Arnone, E., Dudhia, A., Carlotti, M., Castelli, E., von Clarmann, T., Dinelli, B. M., Kleinert, A., Linden, A., Milz, M., Papandrea, E., and Stiller, G.: Impact of temperature field inhomogeneities on the retrieval of atmospheric species from MIPAS IR limb emission spectra, Atmos. Meas. Tech., 3, 1487-1507, doi:10.5194/amt-3-1487-2010, 2010.
Kolonjari, F., Walker, K. A., Boone, C. D., Strahan, S., McLinden, C. A., Manney, G. L., Daffer, W. H., and Bernath, P. F.: ACE-FTS measurements of HCFC-22, Geophys. Res. Abstr., EGU201212440, EGU General Assembly 2012, Vienna, Austria, 2012.

Kyrölä, E., Tamminen, J., Sofieva, V., Bertaux, J. L., Hauchecorne, A., Dalaudier, F., Fussen, D., Vanhellemont, F., Fanton d'Andon, O., Barrot, G., Guirlet, M., Fehr, T., and Saavedra de Miguel, L.: GOMOS $\mathrm{O}_{3}, \mathrm{NO}_{2}$, and $\mathrm{NO}_{3}$ observations in 2002-2008, Atmos. Chem. Phys., 10, 7723-7738, doi:10.5194/acp-10-77232010, 2010.

Laeng, A., Plieninger, J., von Clarmann, T., Grabowski, U., Stiller, G., Eckert, E., Glatthor, N., Haenel, F., Kellmann, S., Kiefer, M., Linden, A., Lossow, S., Deaver, L., Engel, A., Hervig, M., Levin, I., McHugh, M., Noël, S., Toon, G., and Walker, K.: Validation of MIPAS IMK/IAA methane profiles, Atmos. Meas. Tech., 8, 5251-5261, doi:10.5194/amt-8-5251-2015, 2015.

Laube, J. C., Engel, A., Bönisch, H., Möbius, T., Worton, D. R., Sturges, W. T., Grunow, K., and Schmidt, U.: Contribution of very short-lived organic substances to stratospheric chlorine and bromine in the tropics - a case study, Atmos. Chem. Phys., 8, 7325-7334, doi:10.5194/acp-8-7325-2008, 2008.

Mahieu, E., Chipperfield, M. P., Notholt, J., Reddmann, T., Anderson, J., Bernath, P. F., Blumenstock, T., Coffey, M. T., Dhomse, S. S., Feng, W., Franco, B., Froidevaux, L., Griffith, D. W. T., Hannigan, J. W., Hase, F., Hossaini, R., Jones, N. B., Morino, I., Murata, I., Nakajima, H., Palm, M., Paton-Walsh, C., Russell III, J. M., Schneider, M., Servais, C., Smale, D., and Walker, K. A.: Recent Northern Hemisphere stratospheric $\mathrm{HCl}$ increase due to atmospheric circulation changes, Nature, 515, 104-107, 2014.

McDaniel, A. H., Cantrell, C. A., Davidson, J. A., Shetter, R. E., and Calvert, J. G.: The temperature dependent, infrared absorption cross-sections for the chlorofluorocarbons: CFC-11, CFC12, CFC-13, CFC-14, CFC-22, CFC-113, CFC-114, and CFC115, J. Atmos. Chem., 12, 211-227, 1991.

Montzka, S. A., Butler, J. H., Hall, B., Mondeel, D., and Elkins, J. W.: A decline in tropospheric organic bromine, Geophys. Res. Lett., 30, 1826, doi:10.1029/2003GL017745, 2003.

Montzka, S. A., Hall, B. D., and Elkins, J. W.: Accelerated increases observed for Hydrochlorofluorocarbons since 2004 in the global atmosphere, Geophys. Res. Lett., 36, L03804, doi:10.1029/2008GL036475, 2009.

Montzka, S. A., McFarland, M., Andersen, S. O., Miller, B. R., Fahey, D. W., Hall, B. D., Hu, L., Siso, C., and Elkins, J. W.: Recent trends in global emissions of hydrochlorofluorocarbons and hydrofluorocarbons - Reflecting on the 2007 Adjustments to the Montreal Protocol, J. Phys. Chem. A, 119, 4439-4449, doi:10.1021/jp5097376, 2015.

Moore, D. P. and Remedios, J. J.: Growth rates of stratospheric HCFC-22, Atmos. Chem. Phys., 8, 73-82, doi:10.5194/acp-873-2008, 2008.

Murcray, D. G., Bonomo, F. S., Brooks, J. N., Goldman, A., Murcray, F. H., and Williams, W. J.: Detection of fluorocarbons in the stratosphere, Geophys. Res. Lett., 2, 109-112, 1975.

Nedoluha, G. E., Boyd, I. S., Parrish, A., Gomez, R. M., Allen, D. R., Froidevaux, L., Connor, B. J., and Querel, R. R.: Unusual stratospheric ozone anomalies observed in 22 years of measurements from Lauder, New Zealand, Atmos. Chem. Phys., 15, 6817-6826, doi:10.5194/acp-15-6817-2015, 2015. 
Nett, H., Carli, B., Carlotti, M., Dudhia, A., Fischer, H., Flaud, J.M., Perron, G., Raspollini, P., and Ridolfi, M.: MIPAS Ground Processor and Data Products, in: Proc. IEEE 1999 International Geoscience and Remote Sensing Symposium, 28 June2 July 1999, Hamburg, Germany, 1692-1696, 1999.

Nevison, C. D., Kinnison, D. E., and Weiss, R. F.: Stratospheric influences on the tropospheric seasonal cycles of nitrous oxide and chlorofluorocarbons, Geophys. Res. Lett., 31, L20103, doi:10.1029/2004GL020398, 2004.

Nevison, C. D., Dlugokencky, E., Dutton, G., Elkins, J. W., Fraser, P., Hall, B., Krummel, P. B., Langenfelds, R. L., O'Doherty, S., Prinn, R. G., Steele, L. P., and Weiss, R. F.: Exploring causes of interannual variability in the seasonal cycles of tropospheric nitrous oxide, Atmos. Chem. Phys., 11, 3713-3730, doi:10.5194/acp-11-3713-2011, 2011.

Norton, H. and Beer, R.: New apodizing functions for Fourier spectrometry, J. Opt. Soc. Am., 66, 259-264, 1976 (Errata J. Opt. Soc. Am., 67, 419, 1977).

O’Doherty, S., Cunnold, D. M., Manning, A., Miller, B. R., Wang, R. H. J., Krummel, P. B., Fraser, P. J., Simmonds, P. G., McCulloch, A., Weiss, R. F., Salameh, P., Porter, L. W., Prinn, R. G., Huang, J., Sturrock, G., Ryall, D., Derwent, R. G., and Montzka, S. A.: Rapid growth of hydrofluorocarbon 134a and hydrochlorofluorocarbons 141b, 142b, and 22 from Advanced Global Atmospheric Gases Experiment (AGAGE) observations at Cape Grim, Tasmania, and Mace Head, Ireland, J. Geophys. Res., 109, D06310, doi:10.1029/2003JD004277, 2004.

Park, M., Randel, W. J., Kinnison, D. E., Bernath, P. F., Walker, K. A., Boone, C. D., Atlas, E. L., Montzka, S. A., and Wofsy, S. C.: Global Trends of $\mathrm{CHClF}_{2}$ (HCFC-22) and $\mathrm{CCl}_{3} \mathrm{~F}$ (CFC11) estimated from ACE-FTS, HIPPO and WACCM4, SPARC General Assembly 2014, 12-17 January 2014, Queenstown, NZ, 2014.

Ploeger, F., Konopka, P., Müller, R., Fueglistaler, S., Schmidt, T., Manners, J. C., Grooß, J.-U., Günther, G., Forster, P. M., and Riese, M.: Horizontal transport affecting trace gas seasonality in the Tropical Tropopause Layer (TTL), J. Geophys. Res., 117, D09303, doi:10.1029/2011JD017267, 2012.

Prinn, R. G., Weiss, R. F., Fraser, P. J., Simmonds, P. G., Cunnold, D. M., Alyea, F. N., O’Doherty, S., Salameh, P., Miller, B. R., Huang, J., Wang, R. H. J., Hartley, D. E., Harth, C., Steele, L. P., Sturrock, G., Midgley, P. M., and McCulloch, A.: A history of chemically and radiatively important gases in air deduced from ALE/GAGE/AGAGE, J. Geophys. Res., 105, 1775117792, doi:10.1029/2000JD900141, 2000.

Prinn, R. G., Weiss, R. F., Fraser, P. J., Simmonds, P. G., Cunnold, D. M., O'Doherty, S., Salameh, P. K., Porter, L. W., Krummel, P. B., Wang, R. H. J., Miller, B. R., Harth, C., Greally, B. R., Van Woy, F. A., Steele, L. P., Mühle, J., Sturrock, G. A., Alyea, F. N., Huang, J., and Hartley, D. E.: The ALE/GAGE AGAGE Network, Carbon Dioxide Information Analysis Center (CDIAC), Oak Ridge National Laboratory (ORNL, US Department of Energy (DOE), 2013.

Randel, W. J. and Jensen, E. J.: Physical processes in the tropical tropopause layer and their role in a changing climate, Nat. Geosci., 6, 169-176, doi:10.1038/ngeo1733, 2013.

Rasmussen, R. A., Khalil, M. A. K., Penkett, S. A., and Prosser, N. J. D.: $\mathrm{CHClF}_{2}(\mathrm{~F}-22)$ in the Earth's atmosphere, Geophys. Res. Lett., 7, 809-812, doi:10.1029/GL007i010p00809, 1980.
Rinsland, C. P., Boone, C., Nassar, R., Walker, K., Bernath, P., Mahieu, E., Zander, R., McConnell, J. C., and Chiou, L.: Trends of $\mathrm{HF}, \mathrm{HCl}, \mathrm{CCl}_{2} \mathrm{~F}_{2}, \mathrm{CCl}_{3} \mathrm{~F}, \mathrm{CHClF}_{2}$ (HCFC-22), and $\mathrm{SF}_{6}$ in the lower stratosphere from Atmospheric Chemistry Experiment (ACE) and Atmospheric Trace Molecule Spectroscopy (ATMOS) measurements near $30^{\circ} \mathrm{N}$ latitude, Geophys. Res. Lett., 32, L16S03, doi:10.1029/2005GL022415, 2005a.

Rinsland, C. P., Chiou, L. S., Goldman, A., and Wood, S. W.: Long-term trend in $\mathrm{CHF}_{2} \mathrm{Cl}$ (HCFC-22) from high spectral resolution infrared solar absorption measurements and comparison with in situ measurements, J. Quant. Spectrosc. Ra., 90, 367375, doi:10.1016/j.jqstr.2004.04.008, 2005b.

Rodgers, C. D.: Inverse Methods for Atmospheric Sounding: Theory and Practice, Vol. 2 of Series on Atmospheric, Oceanic and Planetary Physics, edited by: Taylor, F. W., World Scientific, Singapore, New Jersey, London, Hong Kong, 2000.

Rothman, L. S., Barbe, A., Benner, D. C., Brown, L. R., CamyPeyret, C., Carleer, M. R., Chance, K., Clerbaux, C., Dana, V., Devi, V. M., Fayt, A., Flaud, J.-M., Gamche, R. R., Goldman, A., Jacquemart, D., Jucks, K. W., Lafferty, W. J., Mandin, J.-Y., Massie, S. T., Nemtchinov, V., Newnham, D. A., Perrin, A., Rinsland, C. P., Schroeder, J., Smith, K. M., Smith, M. A. H., Tang, K., Toth, R. A., Vander Auwera, J., Varanasi, P., and Yoshino, K.: The HITRAN molecular spectroscopic database: edition of 2000 including updates through 2001, J. Quant. Spectrosc. Ra., 82, 5-44, doi:10.1016/S0022-4073(03)00146-8, 2003.

Rothman, L. S., Jacquemart, D., Barbe, A., Benner, D. C., Birk, M., Brown, L. R., Carleer, M. R., Chackerian Jr., C., Chance, K., Coudert, L. H., Dana, V., Devi, V. M., Flaud, J.-M., Gamache, R. R., Goldman, A., Hartmann, J.-M., Jucks, K. W., Maki, A. G., Mandin, J.-Y., Massie, S. T., Orphal, J., Perrin, A., Rinsland, C. P., Smith, M. A. H., Tennyson, J., Tolchenov, R. N., Toth, R. A., Vander Auwera, J., Varanasi, P., and Wagner, G.: The HITRAN 2004 molecular spectroscopic database, J. Quant. Spectrosc. Ra., 96, 139-204, doi:10.1016/j.jqsrt.2004.10.008, 2005.

Saikawa, E., Rigby, M., Prinn, R. G., Montzka, S. A., Miller, B. R., Kuijpers, L. J. M., Fraser, P. J. B., Vollmer, M. K., Saito, T., Yokouchi, Y., Harth, C. M., Mühle, J., Weiss, R. F., Salameh, P. K., Kim, J., Li, S., Park, S., Kim, K.-R., Young, D., O’Doherty, S., Simmonds, P. G., McCulloch, A., Krummel, P. B., Steele, L. P., Lunder, C., Hermansen, O., Maione, M., Arduini, J., Yao, B., Zhou, L. X., Wang, H. J., Elkins, J. W., and Hall, B.: Global and regional emission estimates for HCFC-22, Atmos. Chem. Phys., 12, 10033-10050, doi:10.5194/acp-12-10033-2012, 2012.

SPARC: Report on lifetime of ozone-depleting substances, Their Replacements, and Related Specie, in: WCRP-15/2013, SPARC Report No. 6, edited by: Ko, M. K. W., Newman, P. A., Reimann, S., and Strahan, S. E., WMO/ICSU/IOC, Zürich, 2013.

Steck, T.: Methods for determining regularization for atmospheric retrieval problems, Appl. Optics, 41, 1788-1797, 2002.

Stiller, G. P. (Ed.): The Karlsruhe Optimized and Precise Radiative Transfer Algorithm (KOPRA), vol. FZKA 6487 of Wissenschaftliche Berichte, Forschungszentrum Karlsruhe, Karlsruhe, 2000.

Stiller, G. P., von Clarmann, T., Haenel, F., Funke, B., Glatthor, N., Grabowski, U., Kellmann, S., Kiefer, M., Linden, A., Lossow, S., and López-Puertas, M.: Observed temporal evolution of global mean age of stratospheric air for the 2002 to 2010 period, 
Atmos. Chem. Phys., 12, 3311-3331, doi:10.5194/acp-12-33112012, 2012.

Tikhonov, A.: On the solution of incorrectly stated problems and method of regularization, Dokl. Akad. Nauk. SSSR, 151, 501504, 1963.

Toon, G. C.: The JPL MkIV Interferometer, Opt. Photonics News, 2, 19-21, 1991.

United Nations Environment Programme: Handbook for the Montreal Protocol on Substances that Deplete the Ozone Layer, 8th Edn., Nairobi, Kenya, 2009.

United Nations Environment Programme: Handbook for the Montreal Protocol on Substances that Deplete the Ozone Layer, 9th Edn., Nairobi, Kenya, 2012.

Varanasi, P.: Absorption Spectra of HCFC-22 around $829 \mathrm{~cm}^{-1}$ at Atmospheric Conditions, J. Quant. Spectrosc. Ra., 47, 251-255, doi:10.1016/0022-4073(92)90143-R, 1992.

Varanasi, P., Li, Z., Nemtchinov, V., and Cherukuri, A.: Spectral absorption-coefficient data on HCFC-22 and $\mathrm{SF}_{6}$ for remotesensing applications, J. Quant. Spectrosc. Ra., 52, 323-332, doi:10.1016/0022-4073(94)90162-7, 1994.

Vogel, B., Günther, G., Müller, R., Grooß, J.-U., and Riese, M.: Impact of different Asian source regions on the composition of the Asian monsoon anticyclone and of the extratropical lowermost stratosphere, Atmos. Chem. Phys., 15, 13699-13716, doi:10.5194/acp-15-13699-2015, 2015.

von Clarmann, T.: Validation of remotely sensed profiles of atmospheric state variables: strategies and terminology, Atmos. Chem. Phys., 6, 4311-4320, doi:10.5194/acp-6-4311-2006, 2006.

von Clarmann, T.: Chlorine in the stratosphere, Atmósfera, 26, 415$458,2013$.

von Clarmann, T., Linden, A., Oelhaf, H., Fischer, H., FriedlVallon, F., Piesch, C., Seefeldner, M., Völker, W., Bauer, R., Engel, A., and Schmidt, U.: Determination of the stratospheric organic chlorine budget in the spring arctic vortex from MIPAS B limb emission spectra and air sampling experiments, J. Geophys. Res., 100, 13979-13997, 1995.

von Clarmann, T., Glatthor, N., Grabowski, U., Höpfner, M., Kellmann, S., Kiefer, M., Linden, A., Mengistu Tsidu, G., Milz, M., Steck, T., Stiller, G. P., Wang, D. Y., Fischer, H., Funke, B., Gil-López, S., and López-Puertas, M.: Retrieval of temperature and tangent altitude pointing from limb emission spectra recorded from space by the Michelson Interferometer for Passive Atmospheric Sounding (MIPAS), J. Geophys. Res., 108, 4736, doi:10.1029/2003JD003602, 2003. von Clarmann, T., De Clercq, C., Ridolfi, M., Höpfner, M., and Lambert, J.-C.: The horizontal resolution of MIPAS, Atmos. Meas. Tech., 2, 47-54, doi:10.5194/amt-2-47-2009, 2009a.

von Clarmann, T., Höpfner, M., Kellmann, S., Linden, A., Chauhan, S., Funke, B., Grabowski, U., Glatthor, N., Kiefer, M., Schieferdecker, T., Stiller, G. P., and Versick, S.: Retrieval of temperature, $\mathrm{H}_{2} \mathrm{O}, \mathrm{O}_{3}, \mathrm{HNO}_{3}, \mathrm{CH}_{4}, \mathrm{~N}_{2} \mathrm{O}, \mathrm{ClONO}_{2}$ and $\mathrm{ClO}$ from MIPAS reduced resolution nominal mode limb emission measurements, Atmos. Meas. Tech., 2, 159-175, doi:10.5194/amt-2-159-2009, 2009b.

von Clarmann, T., Stiller, G., Grabowski, U., Eckert, E., and Orphal, J.: Technical Note: Trend estimation from irregularly sampled, correlated data, Atmos. Chem. Phys., 10, 6737-6747, doi:10.5194/acp-10-6737-2010, 2010.

Williams, W. J., Kosters, J. J., Goldman, A., and Murcray, D. G.: Measurements of stratospheric halocarbon distributions using infrared techniques, Geophys. Res. Lett., 3, 379-382, doi:10.1029/GL003i007p00379, 1976.

WMO (World Meteorological Organization): Scientific Assessment of Ozone Depletion: 2014, Global Ozone Research and Monitoring Project - Report No. 55, 416 pp., Geneva, Switzerland, 2014

Xiang, B., Patra, P. K., Montzka, S. A., Miller, S. M., Elkins, J. W., Moore, F. L., Atlas, A. L., Miller, B. R., Weiss, R. F., Prinn, R. G., and Wofsy, S. C.: Global emissions of refrigerants HCFC-22 and HCFC-134a: Unforeseen seasonal contributions, P. Natl. Acad. Sci., 111, 17379-17384, doi:10.1073/pnas.1417372111, 2014.

Yokouchi, Y., Taguchi, S., Saito, T., Tohjima, Y., Tanimoto, H., and Mukai, H.: High frequency measurements of HFCs at a remote site in east Asia and their implications for Chinese emissions, Geophys. Res. Lett., 33, L21814, doi:10.1029/2006GL026403, 2006.

Zander, R., Rinsland, C. P., Farmer, C. B., and Norton, R. H.: Infrared spectroscopic measurements of halogenated source gases in the stratosphere with the ATMOS instrument, J. Geophys. Res., 92, 9836-9850, 1987.

Zander, R., Mahieu, E., Demoulin, P., Duchatelet, P., Servais, C., Roland, G., DelBouille, L., Mazière, M. D., and Rinsland, C. P.: Evolution of a dozen non- $\mathrm{CO}_{2}$ greenhouse gases above central Europe since the mid-1980s, Environ. Sci., 2, 295-303, doi:10.1080/15693430500397152, 2005. 